\title{
Gravity \& Matter Quantum Behaviour from Accelerations, during Electric discharges into Graphite-Based Superconductor
}

\author{
Claude Poher $^{1} \&$ Danielle Poher ${ }^{1}$ \\ ${ }^{1}$ Laboratoire Aurora, Chemin de la Bourdette, Toulouse, France \\ Correspondence: Claude Poher, Laboratoire Aurora, 33 Chemin de la Bourdette, F31400, Toulouse, France. Tel: \\ 33-0-562-177-896. E-mail: claude.poher@wanadoo.fr
}

Received: May 42020

Accepted: May 20, 2020

Online Published: June 30, 2020

doi:10.5539/apr.v12n3p48

URL: http://dx.doi.org/10.5539/apr.v12n3p48

\begin{abstract}
We observe propelling accelerations of a half-kilogram size matter mass, during low energy electric discharges, into a patented "Space Electric Thruster" named "Emitter". This device is made of a Graphite-based, partially superconducting material, at laboratory temperature, and metallic conductors. That emitter propels from theoretically predicted interaction, and emission, of Gravitational Quanta, bearing a momentum, so without ejecting matter particles in Space. From accelerations values measured, with different electric energies, two discharge directions, and three propelling directions, versus the Earth Gravity acceleration direction, we measure the amplitude of several previously unknown phenomena. (i) Quantum nature of Earth Gravity. (ii) Propagation direction of Earth Gravity Quanta. (iii) Expansion of atomic lattice in emitter materials, pushed by Gravitational Quanta. (iiii) Push of free electrons, from the electric powering currents, by Gravitational Quanta. These phenomena confirm predictions from the Inertia and Gravitation model we proposed, and we used to develop our emitters. Same model predicts the observed constant orbital velocity of stars into galaxies, without any Dark Matter mass. And it predicts physical phenomena, observed in our laboratory and published, with another kind of emitter, at cryogenic temperature, during electric discharges.
\end{abstract}

Keywords: Gravitation, Inertia, Space Electric Propulsion, Dark Matter, CDM Models, Superconductors

\section{Introduction}

\subsection{Background}

Our Applied Physics work motivation originated half a century ago, from observations of orbital velocities of matter, into all galaxies of the Universe, by Astronomers (Rubin, 1980). There is an enigmatic behaviour of Gravitation, plus an energetic enigma about the source of the huge Gravitational kinetic energies observed. These enigmas suggested existence of "Dark Matter" mass to many physicists. One of us (CP) was professionally concerned at this time, because being in charge of French Astronomical Space research into the National Institution (CNES). Therefore, we thought, contrarily to Dark Matter hypothesis promoters, that a new Quantum model of Gravitation and of Inertia was required, from which, may be, new propelling devices, for Space technology research, could be imagined, by extracting kinetic energy from Gravitational quanta.

\subsection{Theoretical Model}

Our hypothetic model was built along several decades of private work, and it became theoretically coherent while the 21 th century was beginning. The model justified of course the enigmatic behaviour of Gravitation into galaxies, without any need for Dark Matter mass, which was never observed by fundamental physics institutional laboratories. Our new model required experimental confirmation, of several predicted phenomena, into a laboratory, before being able to be published. These phenomena were theoretically predicted to be created from strong accelerations of elementary matter particles, and the BCS theory of superconductors suggested us, in 2005, the possibility to experiment with a superconducting material.

\subsection{Early Experimental Work}

To begin laboratory verification of our Quantum model, we invented and patented a new kind of Space Electric propelling device, named "emitter" (Poher, 2006 Patent). And we created a private Applied Physics Laboratory (Aurora) for such experiments. Our first emitters were made from YBCO superconductors, in 2007, and they shown immediately all the predicted facts, during electric discharges. We improved these emitters' technology and 
published our experimental results (Poher, 2011, 2012, 2017).

\subsection{Graphite-Based Emitters and New Experimental Possibilities}

Experiments with cryogenic superconductors were made difficult by the required immersion into liquid nitrogen, in order to get the material under its critical temperature. The liquid cooling bath of YBCO emitters was creating artefacts, for accurate measurements of very brief propelling accelerations, during several dozen microseconds, because of its incompressible nature. Then, came the publication of Graphite partially superconducting behaviour, at room temperature (Esquinazi, 1999, 2012, 2014).

We started building new emitters, with Graphite-based material, in 2012, and get efficient propelling results in 2015 , with immersion of emitters into distilled water, at room temperature. We improved that technology and were able to begin experiments without immersion into a cooling liquid, in late 2019. So our 2020 experiments described here, with new efficient Graphite-based emitters, became possible in air, at laboratory temperature, with professional acceleration sensors.

\subsection{Why is Our New Experimentation Important?}

We have experimentally proven that our Quantum model of Inertia and of Gravity, (Poher, 2011, 2012, 2020) can predict experimental behaviour of our emitters into our laboratory. It predicts also galactic velocities without Dark Matter. However, we had not yet observed laboratory experiments where there was an interaction of our emitters with Earth Gravity field. Therefore, Gravitation behaviour was only theoretically modelled. With new experiments made possible in air, thanks to new Graphite-based emitters, and professional accelerometers, we became able to observe the Quantum nature of Earth Gravity. This was required to confirm further our theoretical model.

\subsection{Relevant Scholarship}

Up to now, models of Gravitation, and of Universe evolution (Cosmology models), use Dark Matter hypothesis (Ostriker, 1999 - CERN, 2012 - NASA, 2015), or they use a "MOND" paradigm hypothesis (Modified Newtonian Dynamics, Milgrom, 1983 \& 2020), inserted into interpretations of accurate observations of Cosmological background photons (Collab Plank, 2018). However, there has been not any experimental confirmation of Dark Matter particles observation, in spite of numerous underground laboratories and complex experimental systems.

Therefore, our very simple and easy reproducible experiments, about Quantum behaviour of Earth Gravity, are of fundamental scientific importance.

\subsection{Our Hypotheses and Possible Experimental Confirmations}

There are two required hypotheses for our theoretical model: (i) Existence of a general isotropic flux of Quanta (named "Universons", which differ from Gravitons), propagating at light velocity, into the Universe, (ii) A very short time duration interaction of these Quanta, with elementary massive matter particles, with temporary momentum transfer.

Therefore, direct experiments, involving Earth Gravity, and the possibility to characterize it, are a way to confirm our hypotheses.

\section{Method}

\subsection{Experimental System and Emitters Used}

Our experiments consist to discharge an energy storage capacitor $\mathrm{C}$ into an "Emitter" (EM), which is a superconducting device. The capacitor is first charged at a DC voltage Uo by an external low power DC supply (HV), afterwards it is discharged into the emitter, through a large power thyristor switch (Th). We measure $U o$ and the discharge current $I d$ versus time.

The emitter (EM) is pressed between milled Aluminum electrodes (E+ and E-), bolted to a 623 millimeters long Brass rod, itself bolted on its other end to a calibrated professional miniature piezoelectric accelerometer. We measure the output signal from the accelerometer versus time. The Brass rod introduces a $\approx 200 \mu$ s delay into the acceleration transmitted to the accelerometer. The experimental principle schematic is the following (Figure 1).

The capacitor $(\mathrm{C})$, the power thyristor, and the large Copper bars conducting the current to the emitter are enclosed into an insulated "Power box". The distributed impedance ( $\mathrm{Zi}$ ) of the discharge circuit is composed of a low value distributed Inductance $(\mathrm{L}=2.223 \mu \mathrm{H})$ and a low value variable distributed resistance $(\mathrm{R})$ connected in series. $\mathrm{R}$ includes emitter resistance.

The emitter (EM) is pressed between two milled aluminum electrodes (E+ and E-), having air-cooling fins. Two very flexible electric cables, maintained distant (in order to reduce Ampere forces between the cables), connect the emitter support to the Power box. Flexible cables connections are inverted to get two different directions of the 
discharge current into the emitter, therefore two propelling directions.

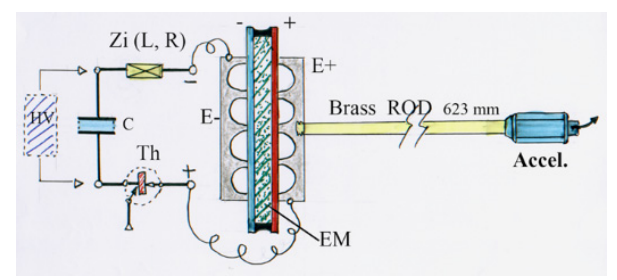

Figure 1. Electric discharges from a storage capacitor are made into a propelling device named "Emitter"

During our experiments, we used successively two different emitters. Emitters composition is detailed into Appendix A. Accelerometer used and its calibration are detailed into Appendix B. The complete experimental system composition is described into Appendix C.

\subsection{Control Experiments and Calibrations}

The first control experiments we have recorded used a stack of two Copper discs instead of an emitter.

Copper is not a superconducting material at $+19^{\circ} \mathrm{C}$, therefore it does not contain Cooper pairs of electrons, only free electrons.

When making an electric discharge into Copper, free electrons are not strongly accelerated, they only move relatively slowly through the "jungle" of atoms, with numerous collisions, captures, and ionizations. No Quantum propelling effect is created.

The role of our control experiments with Copper discs (Dummy emitter) is to make sure there is not any observable set of Ampere forces, acting onto the powering flexible conductors, that could be transmitted to the emitter support, or to the accelerometer. From Appendix E experimental data, we observe that there is not any propelling acceleration measured by the experimental accelerometer. There is only a tiny signal from electromagnetic induction, during the electric discharges of 4.13 and 9.73 Joules ( $U o=$ respectively 200 and 307 Volts), similar to those made later, into a real emitter. See Figures E-1 and E-2 into Appendix E.

Therefore no artefact is observed with that kind of control experiments with Copper discs.

Our next control experiments were about the polarity of the accelerometer signal, onto the oscilloscope screen, versus the acceleration direction applied to the emitter support. This control was simple: a brief mechanical shock was applied manually, onto one of the electrodes of the emitter support, while recording the accelerometer signal. This way, we made clear that the polarity of the first alternation of the accelerometer signal is a real indication of the propelling direction of an emitter. Positive first alternation of the accelerometer signal means acceleration TOWARDS the accelerometer, and the reverse. See Figures E-4, E-5, \& E-6 into Appendix E.

We also observed two facts during these two control experiments:

- We saturated the accelerometer Charge Amplifier output at \pm 10.4 Volts or \pm 104 g's acceleration.

— The accelerometer signal oscillated erratically after each mechanical shock.

With an oscilloscope time window of more than 20 milliseconds width, we observed that the accelerometer signal oscillations, triggered by the mechanical shock, attenuated slowly, in about 15 milliseconds. These oscillations were sinusoidal, with variable amplitude and vertical position.

There are evidently interferences between several harmonic mechanical oscillators into the signal.

— One oscillation with a period of about $625 \mu \mathrm{s}(\approx 16$ kilo Hertz),

— Another oscillation, with a longer period of about $0.4 \mathrm{~ms}(\approx 2,5$ kilo Hertz).

These two resonances frequencies are yet poorly known at that time scale.

\section{The $16 \mathrm{kHz}$ signal is clearly the one of the longitudinal oscillation of the piezoelectric crystal.}

All piezoelectric accelerometers show that same kind of oscillation, during a brief mechanical shock. There is no way to avoid that, as the crystal cannot be damped. The second frequency $(2.5 \mathrm{kHz})$ is the Brass rod resonance.

With an added propelling acceleration, from an oscillating discharge current, a third frequency will be present.

So there are inevitable perturbations to interpret during our experiments.

Nevertheless, by measuring the very first alternation of the oscillating signal, we are able to measure the needed 
peak acceleration value, the one triggering oscillations, for example a propelling effect pulse.

\section{Involving Earth Gravity by Using Three Experimental Configurations}

We want to observe interaction of our emitters propelling effect with Earth Gravity. The propelling effect from our emitters has been proven, since 2011, to be a real Quantum effect, because propulsion is observed without any ejection of matter particles; only emission of Quantum bearers of momentum could be the propelling cause.

Therefore we use here three experimental configurations, by orienting the experimental system of figure 1 into three different directions: (i) Configuration \#1 is with Brass rod vertical, and accelerometer in the Nadir direction, so that Gravity acceleration pushes towards the accelerometer. (ii) Configuration \#2 is with Brass rod horizontal, and Gravity acceleration perpendicular to the sensitivity axis of the accelerometer. (iii) Configuration \#3 is with Brass rod vertical, and accelerometer in the Zenith direction, so Gravity acceleration pushes backwards onto the accelerometer. Configurations details and pictures are shown into Appendix D.

These configurations are not used for measurement of Earth Gravity acceleration, because a miniature piezoelectric accelerometer cannot be used for a constant acceleration. Its Charge Amplifier eliminates any DC signal, up to a frequency of about 10 Hertz. The accelerometer is a differential device, measuring an inertial acceleration, caused by a longitudinal mechanical shock, applied to its stainless steel case.

Therefore we want only to observe the interaction of our emitter with two kinds of Gravitational Quanta: those from the hypothetic general isotropic flux, cause of inertia, and those from Earth Gravity, that our model predicted to have an anisotropic space distribution. We want to check our predictions, AND we want to know the propagation direction of Gravity Quanta.

\section{Results from Our Experiments}

All oscillograms, and accelerations measurements, as well as charge voltages $U o$, and peak discharge currents $I d$, are detailed into Appendix E.

We used first the Graphite-based Emitter EM273, into the three configurations, because of its higher propelling performance. Electric discharges were made at two different energies, from two different charge voltages $U o$, respectively 200 and 300 volts (about 4 and 9 Joules).

And we also observed the same discharges, with an opposed direction of the discharge current. We followed the same procedure for all three configurations. A total of 12 electric discharges were made into emitter EM273.

A typical recorded oscilloscope screen image, of one of these experiments, is reproduced into Figure 2:

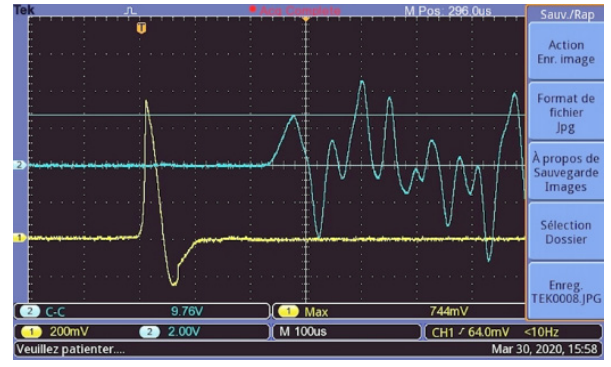

Figure 2. Typical discharge of 204 Volts (4.3 Joules) into EM 273 into configuration Minus 2. Channel \#1 (yellow): Discharge current. Peak value = 3434 Amperes. sepeiChannel \#2 (Blue): Accelerometer signal at $10 \mathrm{~g}$ per Volt $=+27.2 \mathrm{~g}$. Time scale $100 \mu$ s per division

This example is an experiment performed into configuration \#2, with Earth Gravity perpendicular, and a discharge current direction opposed to the direction of the accelerometer, seen from the emitter.

Onto the oscillogram, we observe the time delay, of about $230 \mu$ s, caused by the Brass rod, between the discharge current (yellow curve), and the accelerometer signal (Blue curve).

The peak propelling acceleration value is measured from the oscillogram, thanks to a horizontal cursor, on top of the very first alternation of the accelerometer signal oscillation. This is effectively what triggers the accelerometer oscillating behaviour, after the discharge is finished.

That example shows that the discharge current oscillates, here with a "bizarre" waveform (Abrupt rise).

What is interesting is to synthetize all the experimental results, and compare them. We reproduce here the table of 
results, from Appendix E, with its colors for distinguishing Gravity involvement:

\begin{tabular}{|c|c|c|c|c|c|c|c|}
\hline $\begin{array}{c}\text { Configura- } \\
\text { tion }\end{array}$ & $\begin{array}{c}\text { Polarity } \\
\text { Towards } \\
\text { dccel. }\end{array}$ & $\begin{array}{c}\text { Charge } \\
\text { Voltage Uo } \\
\text { (Volts) }\end{array}$ & $\begin{array}{c}\text { Peak } \\
\text { current Ip } \\
\text { (Amperes) }\end{array}$ & $\begin{array}{c}\text { Peak } \\
\text { Accelera- } \\
\text { tion } \\
\text { (g) }\end{array}$ & $\begin{array}{c}\text { Current } \\
\text { waveform }\end{array}$ & $\begin{array}{c}\text { Direction } \\
\text { of Gravity } \\
\text { +Towards } \\
\text { Accel }\end{array}$ & $\begin{array}{c}\text { Ratio } \\
\text { Ip/ Jo } \\
\text { (Mhos) }\end{array}$ \\
\hline 1 & - & 318 & 2733 & 104 & OK & + & 8.594 \\
\hline 1 & - & 210 & 1662 & 38,4 & OK & + & 7.914 \\
\hline 1 & + & 325 & 2954 & 96 & Neg peak & + & 9.089 \\
\hline 1 & + & 213 & 1809 & 35,2 & Neg peak & + & 8.493 \\
\hline 2 & - & 304 & 3176 & 86 & Neg peak & $\mathbf{0}$ & 10.447 \\
\hline 2 & - & 199 & 1920 & 28 & Neg peak & $\mathbf{0}$ & 9.648 \\
\hline 2 & + & 312 & 5447 & 84 & Abrupt & 0 & $17.458(!)$ \\
\hline 2 & + & 204 & 3434 & 27,2 & Abrupt & 0 & $16.833(!)$ \\
\hline 3 & + & 319 & 2862 & 104 & Neg peak & - & 8.972 \\
\hline 3 & + & 201 & 1754 & 28 & Neg peak & - & 8.726 \\
\hline 3 & - & 316 & 2770 & 98 & OK & - & 8.766 \\
\hline 3 & - & 202 & 1514 & 32,8 & OK & - & 7.495 \\
\hline
\end{tabular}

Figure 3. Results of twelve experiments observed with emitter EM273 for three configurations

The very first observation, made from all the oscillograms, and from the experimental results, is a positive only polarity of ALL accelerations signals, into the twelve oscillograms, an apparent "anomaly".

The second evident "anomalies" are all huge peak accelerations values. As 28 to $104 \mathrm{~g}$ means 274 to $1020 \mathrm{~m} / \mathrm{s}^{2}$ acceleration, for a few Joules of electric energy, a never observed such large value, during past experiments.

Effectively, the propelling direction, and the accelerometer signal, were supposed to revert direction and polarity when changing the discharge current direction, a fact not observed here.

Generally, our Graphite-based emitters have a propelling performance of the order of $2.10^{-4} \mathrm{k} \cdot \mathrm{m} / \mathrm{s}$ per Joule, so about $2.10^{-3} \mathrm{k} . \mathrm{m} / \mathrm{s}$ for 10 Joules electric discharge.

With a discharge current duration of about $100 \mu$ s (Figure 2), the average propelling force of the experimental propelled $0.591 \mathrm{~kg}$ mass, should be about $2.10^{-3} / 100.10^{-6}=20$ Newtons. So the average propelling acceleration value should be about $20 / 0.591=33.8 \mathrm{~m} / \mathrm{s}^{2}$ or $3.45 \mathrm{~g}$, not the ones observed, 28 to $104 \mathrm{~g}$ !

Therefore our measurements reveal the existence of at least another cause, for the observed accelerations. Effectively, from Table 1, we see that the peak acceleration value varies when the configuration is changed.

Moreover, Earth Gravity $(1 \mathrm{~g})$ cannot explain an excess of about $100 \mathrm{~g}$ in the real accelerations values.

\section{Data Interpretation}

It is possible to interpret our experimental results by comparing lines into table 1 . Four phenomena are to be discriminated.

\subsection{Quantum Nature of Earth Gravity}

Let us select, into Table 1, four values of the measured peak acceleration for $\sim 300$ volts discharges.

Exact values of the charge voltages $U o$ of these six discharges are a bit different, into configurations \#1 and \#3 (yellow and green color into table 1), but the peak accelerations values are very close, with a Gravity acceleration oriented parallel to the Brass rod of the accelerometer. These four values are:

$\begin{array}{lllllll}1 & -318 \mathrm{~V} & 104 \mathrm{~g} & + & 325 \mathrm{~V} & 96 \mathrm{~g} & \text { (Gravity on) } \\ 3+319 \mathrm{~V} & 104 \mathrm{~g} & -316 \mathrm{~V} & 98 \mathrm{~g} & \text { (Gravity on) }\end{array}$

Manifestly, a combination of the discharge current direction, with Gravity acceleration direction, has an action.

When the discharge current propagates towards the accelerometer (Polarity + into the table), the propelling acceleration direction should be theoretically in the opposed direction, and inversely.

So the four preceding groups of results, into table 1 can be written:

Propulsion Towards Accelerometer

Propulsion Opposed

Propulsion Opposed

Propulsion Towards Accelerometer

But the Gravity acceleration direction is not the same for each line:

For the fist column, Gravity acceleration is Towards the Accelerometer. For the second column it is the reverse. Therefore, the situations are:

Propulsion Towards Accelerometer + Gravity Propulsion Opposed - Gravity 
Propulsion Opposed + Gravity Propulsion Towards Accelerometer - Gravity

This means that the two discharges of the left column with $104 \mathrm{~g}$ peak acceleration are those where Gravity Quanta ADD to the propelling flux created by our emitter. And it is clearly the reverse for the two discharges of the right column with less acceleration ( 96 and $98 \mathrm{~g}$ ).

This fact proves, without ambiguity, that the two kinds of Quanta (propelling and Gravity) are the same. The propelling effect is proven (Poher, 2011), to be made of Gravitational Quanta, because there is an acceleration of distant matter, and no ejection of matter particles.

Moreover, these facts are $\underline{\text { confirmed }}$ by 300 Volts discharges measurements into configuration \#2 where the Gravity acceleration is perpendicular to the direction of discharge current (yellow part of Table 1):

$2-304 \mathrm{~V}$
$2+312 \mathrm{~V}$

We see here less acceleration value, than when the indication - Gravity applied previously (96 and $98 \mathrm{~g}$ ).

What is Gravity, into our Universons hypothetical model?

It is an anisotropic flux of Universons (Gravitational Quanta). The incident flux, coming from the Zenith direction, is theoretically slightly more intense, than the incident flux coming from the Nadir direction.

And a larger flux, of such quanta, accelerates more matter in the direction of that flux.

An emitter propels by creating an anisotropy, in the distribution of trajectories of emergent Universons, which are natural Quanta, interacting permanently with its superconducting material Cooper pairs of electrons, when these are strongly accelerated. The emergent flux intensity is larger into the direction of the electrons acceleration.

So evidently, an emitter is also sensitive to the intensity of the incident flux of Universons.

The emitter propelling acceleration should therefore be increased, when the incident flux of natural quanta is larger, because of course the emergent flux is proportional to the intensity of incident one.

So, when the discharge current propagates towards the accelerometer (Polarity + ), the propelling flux is emitted also towards the accelerometer, so the propelling acceleration is oriented in the opposed direction of the accelerometer (This is similar to a rocket jet momentum exchange).

Therefore, in configuration $\# 1$, with polarity + , the propelling acceleration is oriented in the minus direction, and it is increased by an increased Gravity flux. This is effectively what is observed.

Therefore Gravity is effectively made of the same kind of Quanta as the ones creating the propelling effect. Gravity is therefore a natural, non-isotropic small intensity flux of these Quanta, which are more numerous, and distributed isotropically, into the Natural flux, we confirmed by previous experiments.

\subsection{Materials Expansion Acceleration}

The Earth Gravity acceleration, added to the propelling acceleration, are not large enough together to explain the very large accelerations values we measured (>100 g). So another physical effect must be involved.

We first tried to explain that strong positive acceleration from a thermal effect dilatation of Graphite, and of metallic electrodes. But the very low electric energies involved were not coherent with that hypothesis.

Nevertheless we made an experiment with Emitter EM265, of a larger mass, to see if the same behaviour was observed. See figures E19 to E23 into Appendix E and the detailed discussion. There is no thermal dilatation.

We also inverted the position of the emitter into its support, to see if the effect was symmetric (Figure E18 and discussion into Appendix E). It is effectively symmetric.

There is no thermal effect, but there is a very fast dilatation (volume expansion) of materials, into the emitter and into the metallic support. This explains why there is always a positive acceleration value, therefore a push towards the accelerometer.

This is not a large expansion. From calculations (relation E1 in Appendix E), $100 \mathrm{~g}$ acceleration corresponds to only 1.57 microns displacement of the metallic electrode facing the accelerometer direction.

Why such an expansion?

We know that the propelling effect is caused by emission of Gravitational Quanta, that are able to accelerate distant matter (Poher, 2011), ant that distant acceleration is inversely proportional to the square of the distance between the emitter and distant matter. 
Therefore, very close to the superconducting zones, into the emitter, that distant acceleration effect should be very large. We supposed therefore that, similarly, emitted Quanta are strongly pushing internal atoms.

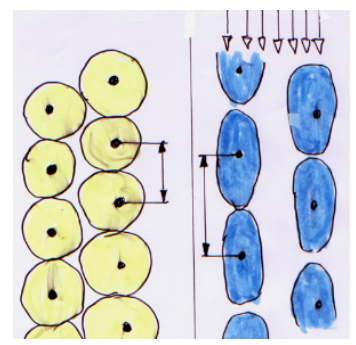

Figure 4. Hypothesis about materials dilatation. On the left is the atomic lattice without propelling Quanta. On the right, propelling Quanta push on all atoms, distorting their shape and expanding inter-atomic distances.

That hypothesis is coherent with experimental results and evolution of acceleration with electric energy.

\subsection{Materials Expansion, Plus Effect of Gravity Flux on Emitter Propelling Effect, Gravity Quanta Direction}

When there is a Gravity field orientation, opposed to the direction of emitter propelling effect, the propelling acceleration subtracts from the dilatation acceleration, so there is less total acceleration measured.

And in configuration \#3, with polarity + , the propelling acceleration is oriented in the minus direction, and it is decreased by an inverse Gravity flux. This reduced propelling acceleration subtracts from the dilatation acceleration, so there is more total acceleration measured.

All experimental results into table 1 confirm these predictions.

So all experimental results say, without any ambiguity, that the incident flux of Gravity quanta, coming from the Zenith direction, is slightly more intense than the flux coming from the Nadir direction. This is undeniable because replicated again and again.

Therefore we know the propagation direction of Gravity Quanta: TOWARDS EARTH CENTER.

This means that Gravity is NOT an attractive effect, but a PUSHING one. Experiments tell the truth.

This is not in accordance with what told Isaac NEWTON centuries ago, this is what tell our model and our experiments.

There is no attraction between masses of matter. There is an Anisotropic flux of Gravity Quanta, because the natural isotropic flux propagation direction seems to be modified by the presence of the Earth mass. This is also compatible with General Relativity (Poher, 2012).

But let us be very careful! This effect cannot be an absorption of Gravity (or propelling Quanta Universons) from the natural isotropic flux, by Earth matter. Because we have already proven, by multiple experiments into our laboratory (Poher, 2011), that there is never any absorption of the propelling Quanta by interposed matter, between an emitter and a distant accelerometer.

According to our theoretical model, matter particles can only change the gravitational Quanta propagation direction, during an interaction. Therefore Gravity can only be a redistribution of the trajectories of Quanta from an isotropic incident flux.

While this is not the subject of this paper, we have theoretically demonstrated (Poher, 2020) that Quantum Fluctuations of the natural isotropic flux of Gravitational Quanta (Universons), are randomly distributed as very brief, intense, and narrow anisotropic "puffs" of Quanta in random directions.

Because of interaction time duration of these Quanta, with matter particles, (which are taking place without average exchange of energy), there is systematically a $4 \pi$ steradians dispersion of these fluctuations "puffs", into Space, at emergence, instead of being anisotropic at incidence.

The apparent result (acceleration), is the same as if there was absorption, except that there is no energy exchange on average. The main isotropic emergent flux, from the Earth direction, is amputated from fluctuations in the Nadir direction. This is not the case from the Zenith direction where there is no distant matter.

Into our experiments, the emitter propelling performance is only modified by Gravity, as theoretically predicted. 


\subsection{Gravity-Dependent Emitter Propelling Performance}

Let us consider again the four preceding 300 Volts discharges (Configurations \#1 and \#3).

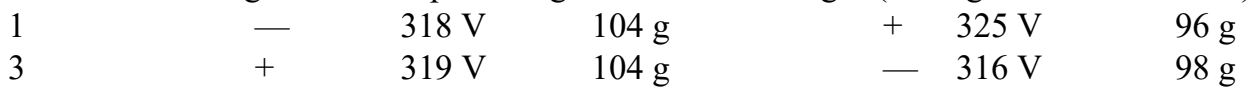

Here, into each horizontal line, the Gravity acceleration direction is the same. But the polarities, meaning the discharge current directions are opposed.

The accelerations difference is $8 \mathrm{~g}$ into the first line and $6 \mathrm{~g}$ into the second line. This means $7 \mathrm{~g}$ average difference. This for two opposed propelling directions, therefore $\pm \mathbf{3 . 5} \mathbf{g}$ into each propelling effect.

We could make theoretical corrections on this value, $\pm 3.5 \mathrm{~g}$, because the $U o$ charge voltages are slightly different $(+2.2 \%$ in first line and $-0.63 \%$ in second line).

Effectively, we have predicted theoretically, and confirmed experimentally, that the propelling momentum of an emitter is proportional to the electric energy of the discharge. So it is proportional to the square of the charge voltage.

But $(325 / 318)^{2}=+4 \%$ correction, and $(318 / 316)^{2}=+1.26 \%$ correction, values that are not large enough to change our conclusions ( $4 \%$ of $3,5 \mathrm{~g}$ is only $0.14 \mathrm{~g}$ ). So we will not make any correction here.

From our experiments, we must consider that the real propelling effect of emitter EM 273, without the emitter dilatation effect, which is not oriented, was really $\pm 3.5 \mathrm{~g}$ for all 300 Volts discharges, or $34.3 \mathrm{~m} / \mathrm{s}^{2}$ for an average stored electric energy of 10.54 Joules.

This corresponds to a total momentum of $2.03 \mathrm{~g} . \mathrm{m} / \mathrm{s}$ of the propelled mass of $0.591 \mathrm{~kg}$.

This is a REAL Propelling Performance of 0.193 g.m/s per Joule (with Gravity).

Now, this is a very classical value for Graphite emitters, confirmed from other kinds of sensors, without contact. So our reasoning seems to be correct.

The preceding value of propelling performance, per powering Joule, is however only valid for configurations \#1 and \#3, where the anisotropic Earth Gravity quantum flux, was parallel to the direction of the discharge current.

In order to know the performance in absence of Gravity effect, we must use the results of configuration \#2 where the Gravity flux was perpendicular to the propelling direction.

Let us recall configuration $\# 2$ results from Table 1 :

$2-304$ Volts $86 \mathrm{~g}$

- 199 Volts $28 \mathrm{~g}$

$2+312$ Volts $84 \mathrm{~g}$

$+\quad 204$ Volts $27.2 \mathrm{~g}$

Without any correction for different charge voltages, it is clear that polarity ( - ) shows always a larger measured acceleration.

The difference between 86 and $84 \mathrm{~g}$ is $2 \mathrm{~g}$ which means a propelling effect of $\pm 1 \mathrm{~g}$ for 300 Volts discharges and 9.79 Joules on average.

And for 200 Volts discharges the same reasoning gives $\pm 0.4 \mathrm{~g}$ propelling effect, for 4.192 Joules on average.

Let us remark that the ratios $\pm 1 / \pm 0.4= \pm \mathbf{2 . 5}$, and $9.79 / 4.192=\mathbf{2 . 3 4}$, are very close to theoretical predictions.

These values of the propelling acceleration for a $0.591 \mathrm{~kg}$ mass gives a:

\section{Propelling Performance without Gravity of 0.102 g.m/s per Joule.}

This is 1.89 times less that with Gravity. Therefore theoretical predictions are confirmed, about the nature, and direction of Quanta, from Earth Gravity.

\subsection{A Strong Effect on the Peak Discharge Current Intensity}

The propelling Universons flux does not only distort wave functions of electrons, in atoms of Graphite and of Aluminum, to show a material expansion. It also extracts electrons from atoms, and it accelerates them in the flux direction, which is also the direction of the electric field acceleration of all free electrons.

Therefore the total number of moving electrons is increased, as well as the total propelling efficiency. The discharge current increases. Its intensity is not only defined from Ohm's Law and the charge voltage Uo.

This fact is clearly observed into our Table 1 results. For this we must look at the right column where the emitter peak conductance is calculated (Ratio: Peak current / Uo).

When a discharge is made through a normal conductor, the conductance is constant when changing the charge voltage. This is simply $\boldsymbol{O h m}$ 's Law. Here, with a superconducting emitter, that conductance is much larger for larger values of Uo. 
This demonstrates that the propelling effect extracts electrons from Graphite.

This effect amplifies therefore the propelling effect (There is a positive reaction).

Several facts are evident from the conductance column of Table 1:

* Conductance is always LARGER during 300 Volts discharges than during 200 Volts.

* Conductance is always LARGER for positive polarities towards the accelerometer, this means when the discharge electronic current propagates towards the accelerometer, as well as the propelling flux itself, according to our theoretical model.

This means when the propelling flux pushes also onto the accelerometer from a distance.

* Conductance is systematically LARGER when the Earth Gravity anisotropic flux is not aligned with the electronic discharge current propagation direction. We checked several times that the two very large values of the conductance in configuration $\# 2$ were reproducible.

Therefore that effect of Gravity acceleration is not at all negligible. The conductance value is doubled for positive polarities towards the accelerometer. This is an indisputable effect.

\subsection{Distortion of the Discharge Current Wave Shape}

During the positive polarities discharges, in configurations \#1 and \#3, as well that during negative polarities discharges, into configuration $\# 2$, there is a negative strong peak at beginning of the discharge current increase. The negative peak amplitude attains $53 \%$ of the peak positive current value.

This phenomenon is never present during discharges into a dummy emitter, made of Copper discs. It is not observed either during discharges into EM 265, where the propelling effect was smaller.

Apparently, this phenomenon is tied to a very strong propelling acceleration, and there seems to be a real negative discharge current, into the thyristor, that would recharge the $206.5 \mu \mathrm{F}$ main capacitor. Effectively the thyristor used can accept very intense reverse currents, as shown into figures 37 to 41 of EM 265 discharges.

During discharges with positive polarities, in configuration \#2 only, the discharge current starting slope is strongly increased, the current attains its peak value in $13 \mu$ s only, instead of $25 \mu$ s normally. It starts rising smoothly, then very abruptly (Figures below).

These effects are clearly caused by the propelling acceleration, not by the dilatation of materials. These are of course caused by intense electrons accelerations into emitter, but remain enigmatic about their distribution.
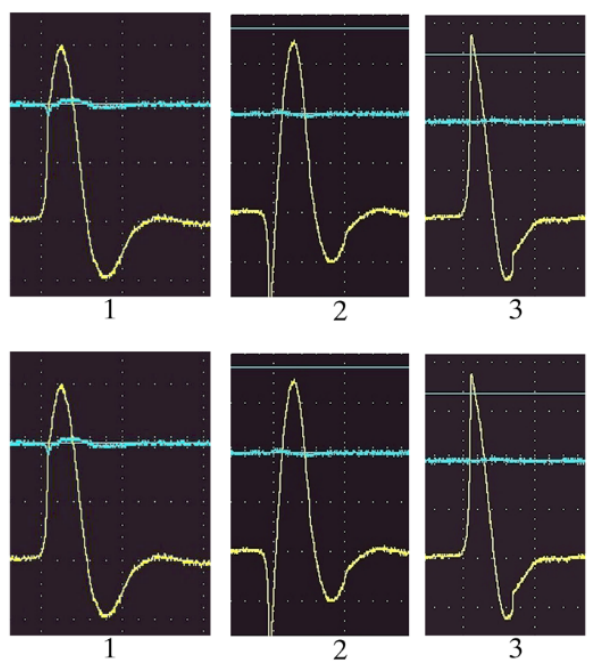

Figure 5. Examples of observed distortions of discharge current wave shapes.

On the left, figure 5.1, a "normal" current wave shape. On the middle figure 5.2, a negative peak distortion, On the right, figure 5.3, a very sharp current increase. These can be observed into many Appendix E figures. They seem being the effect of negative impedance (Energy extraction from the external flux), into the emitter. 


\subsection{Comparison of Propelling Performances of Emitters EM273 and EM265}

Emitter EM273 has 22.3 grams of Graphite with 2mm thickness. Emitter EM265 has 40.9 grams of Graphite with $15.8 \mathrm{~mm}$ thickness. Their thickness ratio $(15.8 / 2=7.9)$ is also the ratio of internal electric fields for a same applied voltage, in favour of EM273.

Their Graphite mass ratio $(40.9 / 22.3=1.83)$ is also the ratio of internal mass of superconducting zones, in favour of EM265. The global, theoretical performance ratio $\left(7.9^{2} / 1.83=34\right)$ is in favour of EM273.

We tested both emitters into configuration \#2, without Gravity effect.

EM273 peak accelerations were 84 and $86 \mathrm{~g}$ for 304 and 312 Volts discharges (10 Joules) with inverse current directions. EM265 peak accelerations were 2.48 and $2.76 \mathrm{~g}$ for 1806 Volts discharges (337 Joules) with inverse current directions. Differences were $2 \mathrm{~g}$ for 10 joules for EM273, versus 1.11g for 337 Joules for EM265.

This means that EM273 Propelling Performance is 60.7 times larger than the one of EM265.

\section{Conclusion, Consequences of These Results}

\subsection{There Is a Propelling Acceleration, the Only Cause of Accelerometer Signal}

It is oriented in the opposed direction of the electronic discharge current into the emitter, as predicted.

The Propelling Performance of the "thin" Graphite emitter EM273 is:

$0.193 \mathrm{~g} . \mathrm{m} / \mathrm{s}$ per Joule with presence of Gravity acceleration.

0.102 g.m/s per Joule without Gravity. The one of "thick" emitter EM265 is 60.7 times lower.

\subsection{There Is a Systematic Dilatation of the Emitters and Support Materials}

This dilatation is manifestly induced by the propelling effect, at atomic lattice scale, into the materials of emitter and of its pressing electrodes, which are very close to the source of propelling effect. This is not a thermal effect. An order of amplitude of that effect seems to be of one micron for a 10 Joules electric discharge, or $0.017 \%$ of materials thickness.

\subsection{There Is a Considerable Increase of the Peak Discharge Current Intensity}

This effect increases with electric discharge energy.

The order of amplitude of that effect seems to be of $8.6 \%$ for a discharge energy increase from 5 to 10 Joules.

There is also a stronger increase of the peak current (1.93 times for a 10 Joules discharge energy), when Earth Gravity acceleration is perpendicular to the propelling direction.

\subsection{There Is a Strong Distortion of the Discharge Current Wave Shape}

The exact and detailed cause of that effect remains enigmatic, particularly its distribution along the three configurations. But only energy extraction, from the natural flux, can be the involved, as only emitters show that.

All these physical phenomena are systematically reproducible, and of a large intensity.

The three configurations we have used here were necessary, to be able to distinguish these effects, and to measure them, in order to determine their causes.

\subsection{More Generally}

All observed experimental phenomena confirm the predictions of our Universons theoretical model of Quantum Inertia and Quantum Gravitation.

Particularly they confirm:

- That our emitters propel themselves during an electric discharge.

- That our emitters propel without anv ejection of matter or of known photons. The emitter is never modified by the propelling effect.

— Of course they MUST propel by emitting invisible Quanta bearing a Momentum.

- Because the propelling momentum is almost proportional to the electric energy of the discharge, these quanta cannot come from the electric energy source, nor from emitter materials, because the propelling kinetic energy is almost proportional to the SQUARE of the discharge electric energy.

— Therefore there MUST be an external source for these propelling Quanta.

- These external Quanta interact manifestly with accelerated electrons inside the superconducting emitter material to create the propelling effect.

— These Quanta MUST BEAR a momentum (or there would not be propulsion).

— Therefore there MUST exist an external flux of these natural Quanta (we named them "Universons").

— That flux of Gravitational Quanta must exist everywhere into the Universe where inertia exists everywhere. 
Our laboratory is not a "special place" for inertia or for Gravity phenomena.

- Earth Gravity acceleration is manifestly also composed of an anisotropic flux of such Quanta, or the Gravity effect we have observed would not exist.

- As Gravity propagates at the speed of light, according to Einstein and to Astronomical events, Gravitation Quanta (Universons) must also propagate at speed of light, everywhere in the Universe.

- These Quanta have all a PUSHING effect (not an attractive one).

— This means they bear a POSITIVE MOMENTUM.

- The main natural flux of Gravitational Quanta MUST BE ISOTROPIC, this means with the same intensity in all directions into the $4 \pi$ steradians of Space, (or matter would be accelerated in the direction of the largest flux). - Earth Gravity Quanta exist into an anisotropic flux, into the direction of the Earth center. The Gravity flux intensity is a little LARGER from the ZENITH than from the NADIR directions (or we would have not observed the Gravity effects).

- But the total natural flux of Gravitational Quanta MUST be much more intense than the Earth Gravity one, because there are huge Astronomical masses, with much stronger Gravity effects. So Earth Gravity effect can only be a tiny part of a HUGE energy global isotropic flux (or we would not have observed hundred of g's acceleration).

- From the energy conservation principle, our emitters EXTRACT kinetic energy from the natural flux (because the propelling momentum is proportional to the electric energy).

- Therefore Humankind has a new huge potential source of energy at its disposal.

- During multiple experiments, published in 2011, we observed that propelling Quanta are never absorbed by matter. And Gravity acceleration is never modified by interposed matter. Therefore Earth Gravity acceleration CANNOT BE CAUSED by absorption of Gravitational Quanta.

- However it can be caused by trajectory deviation of such quanta.

- A Quantum flux is always subject to RANDOM FLUCTUATIONS, it is from these Quantum fluctuations, in intensity and in propagation direction, that there is a Gravity effect. It suffices that trajectories of the fluctuations quanta be modified, to get a pushing acceleration from the external flux, which has not crossed Earth matter.

- We have shown, theoretically, that this can be possible, if time duration of the interaction of Gravitational Quanta, with elementary particles of matter, is LONGER than the average duration of a quantum fluctuation.

These hypotheses are the ones of our Universons Quantum model of Inertia (Poher, 2020).

- Therefore the intensity of the anisotropic component of the Natural flux of Gravitational Quanta, created by macroscopic large masses of matter, becomes smaller at increased distance, as it decreases with the square of the distance (solid angle effect).

This pushing Gravitational flux is made of a randomly fluctuating number of individual quanta, bearing each a same pushing momentum. So distant Gravity momentum value is governed by the average statistical distribution of these quanta.

At solar system Astronomical distances, the statistical distribution of the anisotropic Quanta from the Sun direction follows a Laplace-Gauss distribution, this is the reason why Kepler's orbits Laws apply. (This means the Gravitational acceleration is observed to be inversely proportional to the square of the distance).

But in order to get that proportionality at full solar system scale, the Natural flux from which are coming these fluctuations must have a tremendous intensity.

However at much larger distances between Astronomical masses, such as for most of the stars in Galaxies, the number of anisotropic Universons becomes very small. So the Gauss' distribution cannot apply anymore, it is therefore the Poisson's statistics that must apply.

The average value of a Gauss' distribution and the one of a Poisson's distribution are not at all equal.

So when making real calculations, we observe that, at Galactic range, Gravitational accelerations vary inversely with the distance only (not with its square).

And this should be true for ALL GALAXIES OF THE UNIVERSE.

This is exactly what Astronomers have observed.

So there is no need for any "DARK MATTER" hypothesis.

Our experimental results confirm, as well as our Universons model, that the "Concordance model named Lambda Cold Dark Matter" is not at all an acceptable model, for explaining cosmological behaviour of matter. 
This is a quite strong consequence.

\section{Remarks about Our Accelerations Measurements}

All our experimental oscillograms (Appendix E) show the piezoelectric crystal oscillations triggered by the propelling shock.

These are sinusoidal oscillations, with almost no amortization during the oscilloscope window time duration.

This "harmonic mechanical oscillator" has evidently an associated "over voltage" at its resonance frequency, similar to all oscillators of this kind. We can already see its effect on the right side calibration sheet of our accelerometer, (Appendix B), limited to $10 \mathrm{kHz}$, while the crystal resonance frequency is larger than $10 \mathrm{kHz}$. So we ignore the exact value of the over voltage "coefficient", by which the accelerometer signal amplitude is increased at its resonance frequency.

The discharge current pulses are generally $70 \mu$ s long. Therefore the propelling shocks should have the same duration. Each propelling pulse should correspond, into the frequency domain, to a fundamental frequency component of around 7150 Hertz. There are of course harmonics, with smaller amplitude.

From a Fast Fourier Transform experiment (FFT) of the accelerometer signal (Figure E-24 into Appendix E), we observed that all frequencies over $8 \mathrm{Khz}$ are more strongly attenuated than the $7.5 \mathrm{kHz}$ propelling oscillation. This is also valid for the proper crystal oscillations (33\% attenuation).

Therefore we can consider that our measurement method, based on the amplitude of the very first positive alternation of accelerometer signal, is an acceptable way to measure the experimental total acceleration. At least this method is acceptable to draw the previous conclusions, with a large enough degree of validity.

\section{Acknowledgements}

Our work is private, it is not supported by any institutional organization from our country, or from Europe. We detain full copyright for it. Our acknowledgements are therefore mainly for the members of our families and a few "hidden" friends for their long patience and consensus.

\section{Conflict of interests}

The authors declare that there is no conflict of interests regarding the publication of this paper.

\section{References}

CERN physics, “Dark Matter”. (2012). Retrieved from https://home.cern/science/physics/dark.matter

Josephson. (1974). The discovery of tunnelling supercurrents. Rev. Mod. Phys., 46(2), 251-254. https://doi.org/10.1103/RevModPhys.46.251

Kopelevich, Y., Esquinazi, P., Torres, J. H. S., \& Moehlecke, S. (1999). Ferromagnetic- and Superconductinglike Behavior of Graphite. Retrieved from https://arxiv.org/pdf/cond-mat/9912413.pdf

Milgrom, M. (1983). ApJ, 270, 365. https://doi.org/10.1086/161130

Milgrom, M. (2020). The MOND paradigm. arXiv :0801.3133.

NASA Science Astrophysics. (2015). Dark energy, Dark matter. Retrieved from https://science.nasa.gov/astrophysics/focus-areas/what-is-dark-matter

Ostriker, J. P., \& Steinhardt, P. J. (1999). Cosmic Concordance. Retrieved from https://arxiv:astro-ph/9901388v2

Planck, C. (2018). Plank 2018 results, VI, cosmological parameters.

Poher, C. (2006). European Patent publication WO 2007/093 699 A2, PCT FR 2007/000249. Retrieved from https://www.epo.org/searching-for-patents.html

Poher, C., \& Marquet, P. (2012). General Relativity and Universons. Applied Physics Research, 4(1). https://doi.org/10.5539/apr.v4n1p120

Poher, C., \& Marquet, P. (2012). Universons Gravitation Quantum model in Suppl. Appl. Phys. Res., 4(1). https://doi.org/10.5539/apr.v4n1p120

Poher, C., \& Modanese, G. 2017). Enhanced induction into distant coils by YBCO and silicon-graphte electrodes under large current pulses. Physics Essays, 30, 4. https://doi.org/10.4006/0836-1398-30.4.435

Poher, C., \& Poher, D. (2011). Physical phenomena observed during strong electric discharges into layered Y123 superconducting devices at $77 \mathrm{~K}$. Applied Physics Research, 3(2), 51. https://doi.org/10.5539/apr.v3n2p51

Poher, C., \& Poher, D. (2020). Quantum model of Inertia - Predictions - Confirmations, Consequences for Gravitation into galaxies, and $\Lambda$ CDM Cosmology models. Into publication procedure. 
Riess et al. (2019). Large Magellanic Cloud Cepheid determination of the Hubble's constant. Astrophys. J., 882(1), 34.

Rubin, V., Thonnard, N., \& Ford, W. K. Jr. (1980). Rotational Properties of 21 Sc Galaxies with a Large Range of Luminosities and Radii from NGC $4605(\mathrm{R}=4 \mathrm{kpc})$ to UGC $2885(\mathrm{R}=122 \mathrm{kpc})$. The Astrophysical Journal, 238, 471. https://doi.org/10.1086/158003

Scheike, T., Böhlmann, W., Esquinazi, P., Barzola-Quiquia, J., Ballestar, A., \& Setzer, A. (2012). Can doping graphite trigger room temperature superconductivity? Evidence for granular high-temperature superconductivity in water-treated graphite powder. https://doi.org/10.1002/adma.201202219

\section{Appendix A: Emitters Used During Our Experiments}

\section{A.1 What Is an Emitter?}

We named "emitter" a patented device (Poher, 2006) emitting a Quantum Gravitational Field when crossed by an intense electric current pulse. We described (Poher, 2011) that emission as well as several other physical effects, such as a strong auto-propulsion of the emitter, and acceleration of distant matter, irradiated by the emitted field. Our emitters are always made of a superconducting material, squeezed between two metallic electrodes. Josephson Junctions are implemented into the emitting material (Josephson, 1974).

These Junctions are made by sandwiching a thin layer of a non-superconducting material between two layers of superconducting material. We use grains of insulating material (Silicon carbide, $\mathrm{SiC}$ ), or thin layers of metal as the non-superconducting material into our emitters.

Josephson Junctions role is to get strong local electric fields, where Cooper pairs of electrons can be strongly accelerated during electric discharges. From BCS model of superconductive materials, we predict that electrons can be accelerated into one direction, while the lattice atoms vibrate in a perpendicular plane.

According to our Quantum models of Inertia and of Gravitation, (Poher, 2011, 2020), accelerated electrons interact with natural Quanta from an isotropic Quantum field, in such a manner that a momentum is transferred to emitter matter. Interacting Quanta are then "re-emitted" as a non-isotropic field.

That re-emitted field is a Gravitational field. It accelerates distant irradiated matter.

\section{A.2 Superconducting Material Used}

Several physicists from Leipzig University, (Scheike, Esquinazi \& al., 2012), shown that natural Graphite powder can be made partially superconducting. However only tiny Graphite "islets" of several hundred microns size, are superconducting, with a critical temperature of about $373 \mathrm{~K}$. Therefore only a few $\%$ of that Graphite material mass shows the superconducting behaviour.

We tested fabrication of emitters with Graphite material, and observed that they propelled, with a lower propelling performance than with fully superconducting materials, at cryogenic critical temperature, such as YBCO for example.

However, the high critical temperature of "activated" Graphite allows now experiments without a cooling bath, an interesting simplification, as compared to the use of liquid nitrogen with constraints and artefacts.

According to Leipzig group, the method for developing superconducting tiny zones into graphite powder is:

"Natural Graphite powder is mixed during 24 hours with a hydrogen rich solvent (such as Water, or Alcohol, or Methanol, or Isopropanol)". It seems that Graphite superconducting behaviour is tied to local adsorption of hydrogen molecules into Graphite atomic lattice.

\section{A.3 Two Different Emitters Used}

During experiments described here, we used two different Graphite-based emitters, with a different thickness. This in order to demonstrate internal electric field effect on the intensity of emitted gravitational field.

The two emitters used the same natural Graphite powder with $99.9 \%$ purity, and the same "activation" method with distilled water as the hydrogen source.

\section{A.3.1 Emitter EM 273}

This emitter is made of a $2 \mathrm{~mm}$ thickness Graphite layer with 22.3 grams of Graphite. Its electrodes are made of Aluminum alloy, with a square shape, $100 \mathrm{~mm}$ sides and $2 \mathrm{~mm}$ thickness each. 


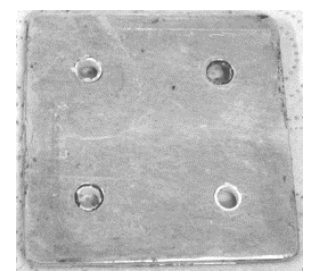

Figure A-1. Emitter EM273, with $100 \mathrm{~mm}$ sides, and $6 \mathrm{~mm}$ total thickness, with $2 \mathrm{~mm}$ Graphite. It has four insulated holes for installation into our experimental system. Graphite powder loss is avoided thanks to a peripheral silicone rubber joint. Total mass is 135 grams, including 22.3 grams of Graphite.

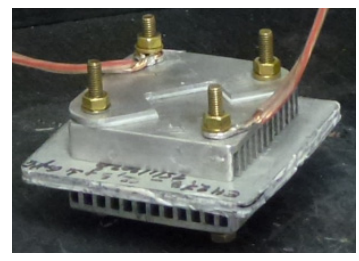

Figure A-2. Emitter EM273 is pressed into an aluminum powering support of the experimental system. This aluminum support has four pressing brass bolts and air-cooling fins. Electric current pulses are sent trough flexible electric cables.

\section{A.3.2 Emitter EM 265}

This emitter is made of a $15.8 \mathrm{~mm}$ thickness layer of Graphite with 40.9 grams of Graphite. Its electrodes are circular, made of Brass, with $60 \mathrm{~mm}$ diameter so $25 \mathrm{~mm}$ total length. There are twelve internal brass disks of 60 $\mathrm{mm}$ diameter and $0.5 \mathrm{~mm}$ thickness, separating the Graphite mass into identical layers. Its periphery is made of silicone joint reinforced by glass epoxy tape. Its total mass is 255 grams.

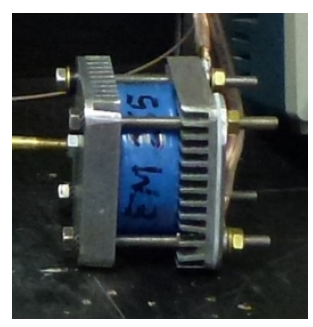

Figure A-3. Emitter EM 265 installed into the same pressing and powering support.

\section{A.3.3 Functional Differences between EM273 and EM265}

Metallic electrodes nature and mass have no effect onto the propelling performance observed. Only the superconducting mass and the internal electric fields drive the performance.

Emitter EM265 has 1.834 times more Graphite mass than EM273. And EM273 has 7.9 times less thickness of Graphite material than EM265.

We can suppose that the average total mass of superconducting zones into Graphite is proportional to the Graphite total mass. So EM265 should have 1.834 times more superconducting mass inside than EM273.

About internal electric fields, we can expect them to be inversely proportional to the graphite total thickness for a same applied external voltage.

Therefore, there should be 7.9 times more electric field intensity inside EM273.

Consequently, emitter EM273 should be theoretically about $7.9^{2} / 1.834=34$ times more efficient than EM265. This even if their total mass ratio is inversely equal to $135 / 255=0.53$.

\section{A.4 Dummy (Control) Device}

We also make similar electric discharges into a device having no Graphite into it. This device, used for control, is made of two metallic conductive electrodes. Without a superconductor, it does not propel, and does not emit any 
Gravitational field.

The control device we use is made of two Copper discs pressed together, with a diameter of $60 \mathrm{~mm}$ and $3 \mathrm{~mm}$ thickness each. This way, the total thickness is $6 \mathrm{~mm}$, similar to the one of EM 273.

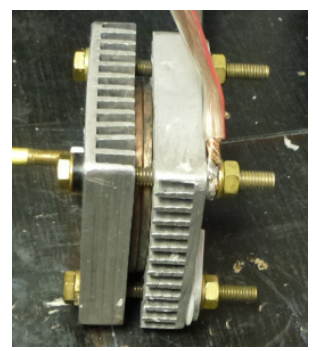

Figure A-4. "Dummy" (control) emitter made of two Copper discs, installed into the same pressing and powering support

We use that control device for comparison of results.

\section{Appendix B: Propelling Effect Measuring Instrument}

\section{B.1 Very Short Duration Experiments}

During all our experiments, we make electric discharges into different emitters. These create a propelling effect, from the acceleration of electrons by an electric field. There is a momentum transferred to the emitter, and to its metallic support. The propelling momentum total duration is the one of the electric current (Poher, 2011).

To be observed easily, the propelling momentum creation requires an applied electric voltage to emitters of at least several hundred Volts. The devices being made of a superconducting material, of a very low electric resistance, the current pulse sent to emitters has an intensity of several thousands Amperes.

We want to avoid the difficulties from a liquid cooling bath, this means we only use here natural air cooling.

So we cannot use thousands of Amperes and hundred of volts (megawatts of electric power), for a long time, or the critical temperature of the superconducting material would be exceeded.

Therefore all our experiments are using low energy electric discharges, 5 to 10 Joules, avoiding a temperature increase of internal Graphite. Of course the electric power is still of the order of megawatts, but the current pulses duration is very short, of the order of 100 to 150 microseconds.

\section{B.2 Piezoelectric Accelerometer}

Measuring a propelling effect of several dozen microseconds duration, is only possible with a fast accelerometer. There are no other instruments existing for that purpose. There are professional miniature calibrated piezoelectric accelerometers able to measure accelerations of 150 microseconds duration and less.

We used an industrial miniature accelerometer of that type, made by DJB instruments in UK, capable of $110 \mathrm{~g}$.

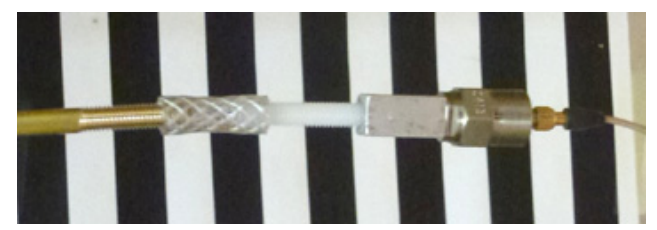

Figure B-1. Miniature accelerometer DJB \# 5413 bolted at end of a brass rod with insertion of an insulating separation made of fiberglass. The accelerometer itself is the stainless steel "nut" on the right (12 mm diameter)

Such accelerometers contain a tiny piezoelectric crystal (made of Quartz for example), with gold plated electrodes. One of these electrodes is soldered to the stainless steel enclosure. The other electrode is connected to a "Charge Amplifier" by a coaxial cable. When a shock is applied to the accelerometer, the crystal inertia presses the material, and an electric charge appears between its two electrodes.

Electrically, the accelerometer is a tiny value capacitor, because its crystal is a dielectric. Mechanically, this is a "Harmonic oscillator", like a mass suspended to a spring. The mass is the one of the crystal, and the spring is the crystal stiffness. It has a single, longitudinal, direction of sensitivity. 
This means that all miniature accelerometers have a resonance oscillation frequency. Therefore, similarly as a suspended mass, the crystal electric signal (charge) is proportional to the stainless steel enclosure acceleration, only for frequencies under the resonance.

At resonance, the crystal oscillation amplitude becomes larger than the enclosure one. And over the resonance frequency, there is a crystal oscillation amplitude attenuation of 6 decibels per octave of frequency increase, and there is a phase shift of $\pi$ radians (phase inversion).

Therefore miniature piezoelectric accelerometers should normally be used under their resonance frequency.

Accelerometer DJB \# 5413 has a resonance frequency larger than $15 \mathrm{kHz}$.

An emitter propelling momentum, with a total duration of 150 microseconds, is a brief mechanical shock, which corresponds to a fundamental frequency of $6.67 \mathrm{kHz}$, with harmonics. This is under accelerometer resonance.

Under its resonance frequency, a piezoelectric accelerometer is a very linear instrument, very reproducible, and can be accurately calibrated. But brief shocks force it to oscillate after the shock is finished.

\section{B.3 Accelerometer Charge Amplifier, and Calibration}

It is not possible to connect a miniature piezoelectric accelerometer directly to the input of an oscilloscope, for measuring accelerations. Effectively the signal from the accelerometer would be strongly attenuated, and its wave shape distorted. The cause lays into the very large impedance of the piezoelectric crystal.

Therefore, a specific electronic amplifier (named a Charge Amplifier) must be inserted between the accelerometer and the oscilloscope input.

This is a very large input impedance amplifier (hundreds of megohms), with a constant gain from 10 Hertz to hundreds of kilo Hertz. And this is a calibrated amplifier.

Such Charge Amplifiers are sold with the accelerometer, and calibrated with it.

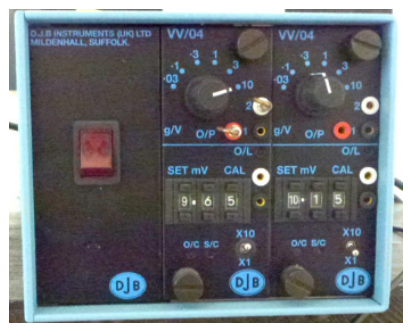

Figure B-2. Double DJB charge amplifier front panel, with calibrated switches

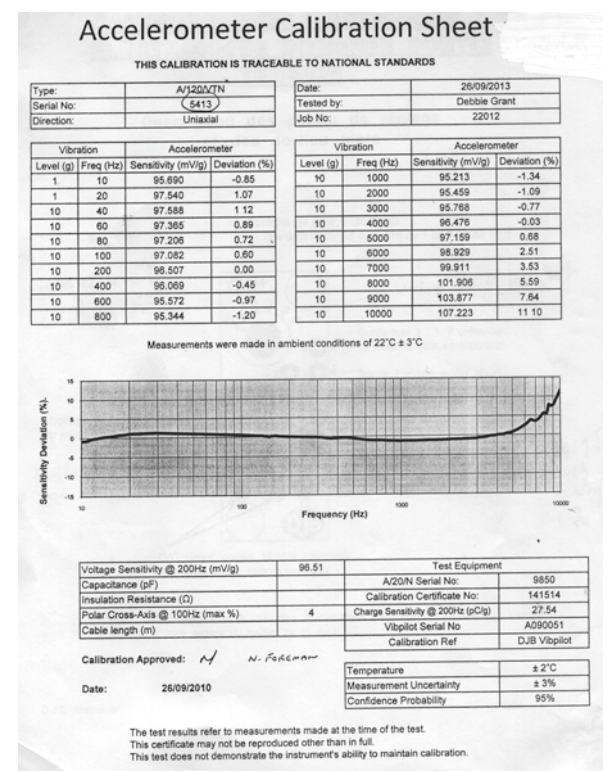

Figure B-3 - Calibration data for accelerometer \# 5413 
We use the left side amplifier, calibrated for accelerometer \# 5413, thanks to three coding wheels, and a lower switch (X10 or X1). Into the coding wheels window are entered, from the wheels, and from the switch, the accelerometer sensitivity value, expressed in millivolts per $\mathrm{g}$ at a frequency of 200 Hertz. One $\mathrm{g}$ acceleration is $9.81 \mathrm{~m} / \mathrm{s}^{2}$ in international units. So here we entered $96.5 \mathrm{mV}$ per g. This is the calibration method.

The calibration parameter is known from a calibration sheet, delivered for each accelerometer (see Figure B-3).

Accelerometers are calibrated onto a specific installation, where sinusoidal mechanical oscillations of a piloted peak amplitude are applied to the accelerometer. The output signal from the piezoelectric crystal, connected to its amplifier, is an $\mathrm{AC}$ sinusoidal voltage, with the same frequency as the input oscillation, and a maximum amplitude measured and reported onto the calibration sheet. This is done for frequencies from 10 to $10000 \mathrm{Hertz}$.

From that calibration sheet we know that the response is flat $\pm 2 \%$ from 10 Hertz up to 6000 Hertz. Then it climbs to $+11 \%$ at $10 \mathrm{kHz}$. The resonance frequency is larger than $10 \mathrm{kHz}$. Sensitivity at 200 Hertz is $96.51 \mathrm{mV}$ per g, and it is almost the same all along the spectrum.

The Charge Amplifier has an upper rotating commutation switch for choosing its gain. This gain can be set from 0.03 to $10 \mathrm{~g}$ 's per output Volt.

The amplifier saturates for about \pm 11 Volts, therefore we can measure up to $110 \mathrm{~g}$ 's peak acceleration.

This is a good, accurate, reproducible, experimental sensor, with specific resonance oscillation as will be shown.

\section{B.4 Electromagnetic Induction Protection, Long Brass Rod}

A piezoelectric accelerometer is sensitive to the surrounding electromagnetic field. A voltage is induced into the piezoelectric crystal capacitor by the field. A "charge displacement field" $D$ appears in dielectrics, according to Gauss' law:

$$
\Delta . D=\rho f
$$

Where $\rho f$ is the number of free charges per unit volume of dielectric. The charge displacement field $D$ is equal to the induced electric field $E$ times the permittivity $\varepsilon$ of the dielectric. So the induced voltage $V$ between two electrodes separated by a distance $d$ into a dielectric of permittivity $\varepsilon$ is:

$$
V=D d / \varepsilon
$$

The discharge current of several thousands amperes, into our emitters, creates a strong electromagnetic field during the 100 to 150 microseconds of electric powering pulse. And it is precisely during that current that there is a propelling effect we want to measure with the accelerometer.

If the accelerometer was directly bolted onto the emitter support, (Figures A-2 and A-3), it would have sensed simultaneously the propelling mechanical shock pulse, plus the electromagnetic field.

The output signal from an accelerometer is always the sum of two effects: acceleration signal, plus electromagnetic induction signal. If the two were simultaneous, there would be no way to separate the two signals. There is some attenuation of electromagnetic fields by the stainless steel case of an accelerometer, which is a kind of tiny Faraday's cage. But for our experiments this is not sufficient.

Therefore we used another method, thanks to a $\mathbf{6 2 3} \mathbf{~ m m ~ l o n g ~ B r a s s ~ r o d ~ i n s e r t e d ~ b e t w e e n ~ t h e ~ e m i t t e r ~ s u p p o r t , ~ a n d ~}$ the accelerometer.

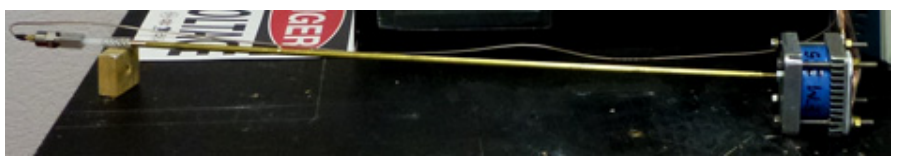

Figure B-4. The accelerometer is bolted at one end of a $623 \mathrm{~mm}$ long Brass rod of $6 \mathrm{~mm}$ diameter. The other end of the rod is bolted onto the emitter support. The rod introduces $\mathrm{a} \approx 190 \mu$ s delay

The mechanical propelling momentum propagates to the accelerometer, almost without attenuation, at the speed of sound into Brass (about $3300 \mathrm{~m} / \mathrm{s}$ ), while the electromagnetic field propagates at speed c $=3.10^{8} \mathrm{~m} / \mathrm{s}$. This means that the rod brings a propagation time delay, for the propelling momentum, which is chosen longer than the electric current pulse duration. With a $623 \mathrm{~mm}$ long rod, the delay is about $190 \mu \mathrm{s}$ while the electric pulse duration is less than $150 \mu \mathrm{s}$. 
This way there is no more mixing of the two output signals, from the accelerometer. An oscilloscope is therefore able to discriminate the two effects by their different timing.

\section{B. 5 Propelled Mass}

During each electric pulse, our emitters create a momentum, inside the superconducting zones of the Graphite material. This momentum is transferred, by interactions between adjacent atoms, to the whole mass of materials strongly attached to the emitter. The total propelled mass is composed of: the emitter itself (135 gram for EM 273, or 255 grams for EM265), the emitter support, the Brass rod, and the accelerometer.

\section{The total propelled mass is 591 grams with emitter EM273 and it is 711 grams with emitter EM265.}

\section{B.6 Propelling Effect Acceleration}

We observed experimentally and predicted theoretically (Poher, 2011) that all our emitters create a propelling momentum, proportional to the electric energy sent to them.

Graphite based emitters, of the size we use here, have a propelling performance of about $2.10^{-4} \mathrm{~kg} . \mathrm{m} / \mathrm{s}$ per electric Joule. We intend to send them about 10 Joules maximum of electric energy.

Therefore a total propelling momentum of about $2 \cdot 10^{-3} \mathrm{~kg} \cdot \mathrm{m} / \mathrm{s}$ is expected, during the 100 to 150 microseconds of electric powering.

This corresponds to a maximum average pushing force of $2.10^{-3} / 100.10^{-6}=20$ Newton. And the acceleration of a 591 grams mass should be $A=20.0 .591=33.84 \mathrm{~m} / \mathrm{s}^{2}$ or $3.45 \mathrm{~g}$.

Therefore the chosen accelerometer, and its Charge Amplifier, are adapted to this measurement.

\section{Appendix C: Description of Our Expermental System}

\section{C.1 Principle of Our Experiments}

Our experiments consist to send electric energy to an emitter, and observe the propelling acceleration. We want to measure the total electric energy, the electric current evolution into the emitter, and the propelling acceleration evolution with an accelerometer. Therefore an experiment electric diagram is the following:

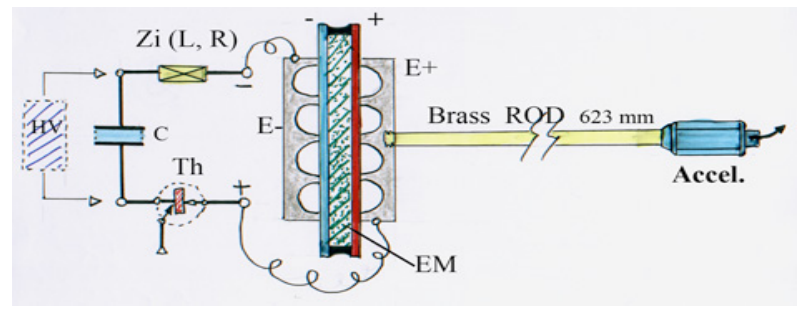

Figure C-1. Experiment principle with storage capacitor C, emitter E, Brass rod, and accelerometer

During all our experiments, we discharge an energy storage capacitor C into an "Emitter" EM. The capacitor is first charged at a DC voltage Uo by an external low power DC supply (HV). Then it is discharged into the emitter through a large power thyristor switch (Th). We measure $U o$ and the discharge current $I d$ versus time.

The emitter (EM) is pressed between electrodes (E+ and E-) bolted to a 623 millimeters long brass rod, bolted itself on its other end to a calibrated professional miniature piezoelectric accelerometer. We measure the output signal from the accelerometer versus time. The brass rod introduces $\mathrm{a} \approx 190 \mu$ s delay into the acceleration transmitted to the accelerometer with negligible attenuation.

The capacitor $(\mathrm{C})$, the power thyristor, and the large copper bars conducting the current to the emitter, are enclosed into an insulated "Power box". The total distributed impedance ( $\mathrm{Zi})$ of the discharge circuit, is composed of a low value distributed Inductance $(\mathrm{L}=2.223 \mu \mathrm{H})$, and of a low value variable distributed resistance $(\mathrm{R})$, both connected in series. $\mathrm{R}$ includes emitter resistance.

The emitter (EM) is pressed between two milled aluminum electrodes (E+ and E-), with air cooling fins. Two very flexible electric cables, maintained distant, connect the emitter support to the Power box, in order to reduce Ampere forces between the cables, to be transmitted to the emitter.

The flexible cables connections can be inverted, to get two different directions of the discharge current into the emitter.

The discharge current $I d$ is measured by an oscilloscope connected to a "Rogowski" coil sensor. 


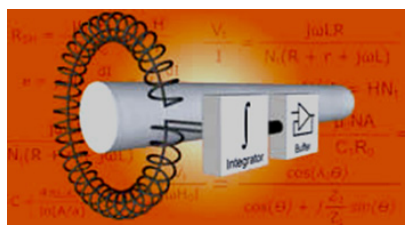

Figure C-2. Principle of a Rogoswski coil sensor for measuring a variable current without contact

A Rogowski coil sensor is a device invented by German physicist Walter Rogowski, to measure variable electric currents, without contact with the conductor where the current circulates. It is composed of a flexible coil surrounding the main conductor, and an integrator / amplifier circuit. The voltage induced into the coil is proportional to the main current derivative, so after integration, the integrator output signal is proportional to the main current. Such sensors can measure accurately currents of a very large intensity, at a frequency larger than 100 $\mathrm{kHz}$. They must be calibrated by laboratory methods.

Our experimental, passive integrator Rogowski sensor, delivers 4.616 millivolts per Ampere current; it was calibrated from a professional Rogowski current probe MA200, from Chauvin-Arnoux, with an active integrator limited to 3000 Amperes peak, because of its internal 9 Volts battery. Our sensor is limited to 30000 Amperes.

\section{C.2 Experimental System Composition}

The full experimental system is made of: the Power box, an emitter with its support, and brass rod, a professional miniature calibrated accelerometer, a calibrated Charge Amplifier for the accelerometer, two flexible cables to connect the emitter support to the power box, a two channels oscilloscope, a low power supply DC high voltage.

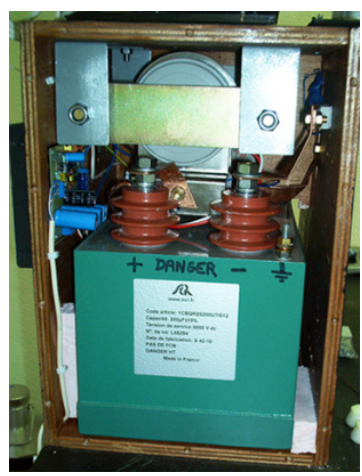

Figure C-3. Power box. The Energy storage capacitor C of $206.5 \mu \mathrm{F}$ value is the large green lower volume. It is insulated to 5000 Volts. The power thyristor (Dynex PT85) is installed over it. Insulated to 3000 Volts, it is capable of $20 \mathrm{k}$ Amperes discharge current. On the left side is the electronic / optical relay for triggering the thyristor, from a safe distance, by wire manual command. Components are connected by $50 \mathrm{~mm}^{2}$ Copper bars. A Rogowski coil sensor plus its passive integrator allow the discharge current measurement. On the front panel

(right side) is the four digits calibrated voltmeter, measuring the charge voltage Uo of capacitor $\mathrm{C}$

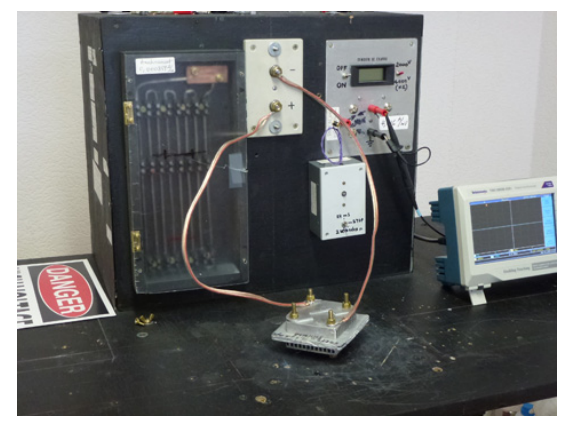

Figure C-4. Emitters are connected to the Power box by two very flexible cables, maintained as distant as possible, to reduce Ampere forces between them. Inverting the connections of these cables allows inversion of the discharge current direction into an emitter. On the right side, onto the Power box, is the digital voltmeter to measure the charge voltage Uo. The two Rogowski sensor outputs are situated under this voltmeter 


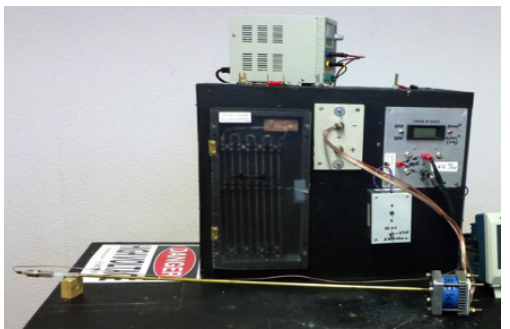

Figure C-5. The 6000 Volts high voltage DC power supply is the grey box, put down on top of the Power box

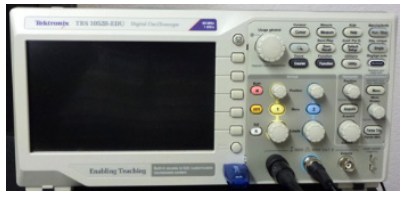

Figure C-6. Experimental results are observed onto a digital oscilloscope Tektronix Model TBS 1052B. It has two channels, Dc to $40 \mathrm{Mhz}$ max frequency, and $1 \mathrm{GHz}$ sample rate. Screen images are recorded on a USB key. Channel \#1 is connected to the Rogowski current sensor, with 4.616 Amperes per millivolt calibration factor. Channel \#2 is connected to the output of the accelerometer Charge Amplifier with an adjustable calibration. Oscilloscope triggering is generally done by channel \#1 (current), when signal is larger than a chosen value

\section{C.3 Discharge Energy and Discharge Current Oscillation}

Stored electric energy Eo is known from the measurement of charge voltage Uo, and capacitor $\mathrm{C}$ value.

$$
\mathrm{Eo}=1 / 2 \mathrm{C} \mathrm{Uo}^{2}
$$

With $C=206.5 \mu \mathrm{F}, E o=4.13$ Joules when $U o=200$ Volts, and $E o=9.29$ Joules when $U o=300$ Volts.

These are the experimental energies we use during the experiments reported here. Such low value energies avoid any thermal increase of the Graphite temperature, which remains the one of the laboratory atmosphere $\left(+19^{\circ}\right.$ C).

We checked the emitter temperature with a digital thermometer, and never observed any variation, thanks to the emitter support thermal inertia and its air-cooling fins.

Moreover, energy Ee, which is really transferred to the emitter, is lower than the stored electric energy Eo, because the Power box circuit low total impedance is almost equal to the emitter low internal resistance. Therefore the emitter receives only 40 to $50 \%$ the stored electric energy Eo. This means 2 to 5 Joules only.

The discharge current oscillates, because the total dissipating resistance R of the discharge circuit (Figure C-1) is lower than the critical, non-oscillating condition:

$$
\mathrm{R} \geq 2(\mathrm{~L} / \mathrm{C})^{1 / 2}
$$

With a distributed inductance $L=2.223 \mu \mathrm{H}$ and a capacitor value $C=206.5 \mu \mathrm{F}$, the total resistance $R$ value should be larger than $0.208 \mathrm{Ohm}$ to avoid oscillations.

But the real total resistance of the circuit, emitter included, is generally smaller than this limit. So the current oscillates with a rapid amortization.

The oscillation frequency Fo of the discharge current is equal to:

$$
\mathrm{Fo}=\left(2 \pi(\mathrm{L} \mathrm{C})^{1 / 2}\right)^{-1}=7428 \mathrm{Hertz}
$$

\section{Appendix D: Three Experimental Configurations}

\section{D.1 Introduction}

We want to measure the propelling effect acceleration with an accelerometer, for two levels of the stored electric energy, of about 4 and 9 Joules. This corresponds respectively to $U o=200$ Volts and 300 Volts.

Moreover we want to observe the propelling momentum direction versus the direction of the discharge current into the emitter; because, theoretically, that propelling direction should be opposed to the current direction of electrons acceleration. We can change the current direction by inverting the connections of the flexible electric cables to the Power box. 
And we want to do that for two different emitters, EM273 and EM265.

So we have two values of the voltages $U o$ (200 and 300 Volts), two current directions, and two emitters. This requires 8 electric discharges.

Moreover, we need to observe two control experiments, to know the accelerometer signal polarity, versus the acceleration direction. These are not electric discharges, only mechanical shocks.

And there are also two control discharges into Copper discs (dummy emitter) at 200 and 300 Volts.

That corresponds to a total of 12 oscillograms. All these can be made with the same experimental configuration.

\section{D.2 Intervention of Earth Gravity Direction}

We perform experiments with emitters creating a gravitational field. Earth Gravity field is also a Gravitational field.

Therefore we want to observe what happens, when the Earth Gravity acceleration direction is oriented parallel, perpendicular, or opposed, to the direction of the emitter gravitational field.

We cannot change the vertical direction of the Earth Gravity, but we can change the direction of the emitter Gravitational field. Theoretically, this field is oriented in the direction of electrons acceleration into the Graphite layer of an emitter.

This means that we need to observe the propelling acceleration, for three orientations of the propelled mass.

Configuration 1 will be a VERTICAL orientation of the propelled mass, with accelerometer in the $\underline{\text { NADIR }}$ direction.

Configuration 2 will be a HORIZONTAL orientation of the propelled mass.

Configuration 3 will be a $\underline{V E R T I C A L}$ orientation of the propelled mass, with accelerometer in the $\underline{Z E N I T H}$ direction.

With two voltages $U o$, two directions of the discharge current, and three configurations, we need to record 12 oscillograms for one emitter. We are not going to change the configuration for the two emitters, only for emitter EM273. Comparative discharges into EM265 and into the Copper discs will be observed in configuration 2 only, where Earth Gravity does not have any effect. The same configuration 2 will be used for the mechanical polarity controls shocks.

Nevertheless even with these simplifications, we need to observe a total of 20 oscillograms.

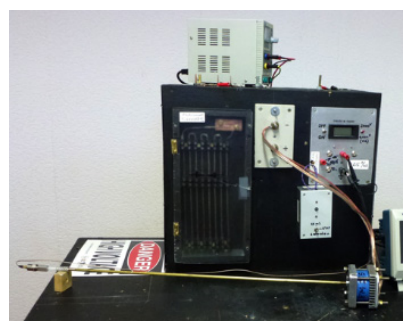

Figure D-1. Configuration \#2 experiments, with emitter EM265. Brass rod is horizontal

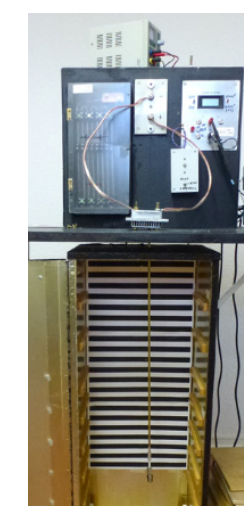

Figure D-2 - Configuration \#1 experiments, with emitter EM273. Brass rod is Vertical, accelerometer is in the NADIR direction. Gravity acceleration is oriented towards the accelerometer direction 


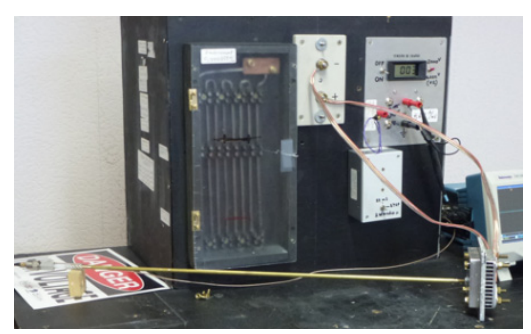

Figure D-3. Configuration \#2 experiments, with emitter EM273. Brass rod is Horizontal. Gravity acceleration is PERPENDICULAR to the accelerometer direction

These three configurations are not chosen for detection of Earth Gravity acceleration by the accelerometer, because this instrument cannot detect the Gravity acceleration.

First because this is a constant acceleration value, and the Change Amplifier does not transmit a DC signal. Moreover, because an accelerometer detects only a differential push, onto its piezoelectric crystal.

When the crystal, and the stainless steel case, are submitted to a same gravitational field, there is no differential effect, therefore there is no acceleration signal output from the crystal. A pressure must be exerted onto the extremities of the piezoelectric crystal to get an output charge. With common accelerations, no charge exists.

When the emitter propels, it pushes only the stainless steel case of the accelerometer, by the Brass rod. So by inertia of the crystal mass, there is pressure onto the crystal faces, and an output electric charge.

Therefore what we want to observe is the effect of Earth Gravity field onto the emitter functioning, not onto the one of the accelerometer.

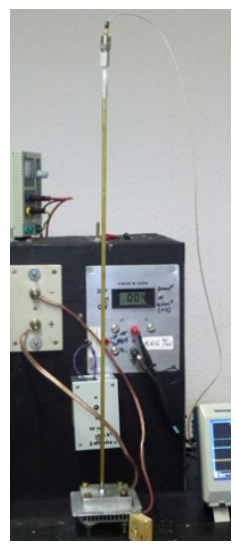

Figure D-4. Configuration \#3 experiments with emitter EM273. Brass rod is Vertical, accelerometer is in the ZENITH direction. Gravity acceleration is oriented towards the emitter direction

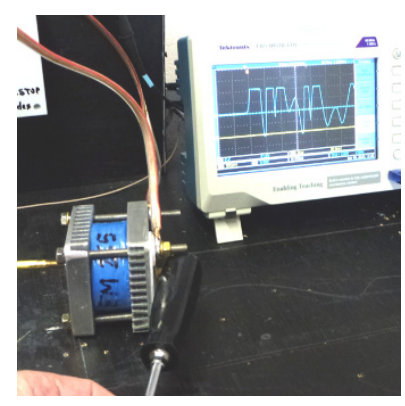

Figure D-5. Configuration \#2 experiment with emitter EM265 and a mechanical shock, oriented towards the accelerometer, for control of the accelerometer signal polarity (here polarity is POSITIVE on oscilloscope) 


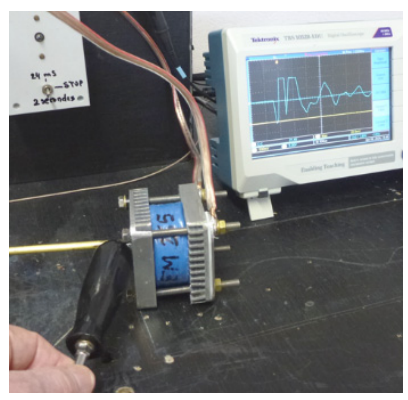

Figure D-6. Configuration \#2 experiment with emitter EM265 and a mechanical shock, oriented opposed to the accelerometer, for control of the accelerometer signal polarity (here polarity is NEGATIVE on oscilloscope). The "polarity" is the sign of the signal for the first alternation of the accelerometer signal. This way we should be able to know the direction of the propelling effect

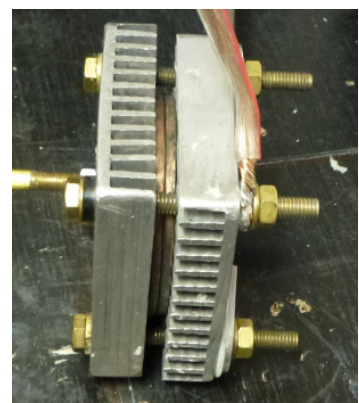

Figure D-7. Configuration \#2 CONTROL experiments, with “dummy” emitter made of Copper discs

Control experiments with mechanical shocks onto the emitter support do not need any emitter, nor a discharge, we made them with emitter EM265 installed into its support and in configuration \#2.

Control experiments with discharges into Copper discs, do not require to change the current direction, because there is not any propelling effect created, they were performed in configuration $\# 2$.

\section{Appendix E: Experimental Data}

\section{E.1 Data Recording, and Oscilloscope Screen Images}

During experiments, the charge voltage $U o$ of capacitor $C$, is manually chosen, by looking at the digital voltmeter situated onto the Power box. It is difficult to get exactly 200 or exactly 300 Volts manually, so the voltage $U o$ values change a little bit from one discharge to the next. The exact value indicated by the voltmeter is recorded manually, onto a portable computer, immediately after a discharge.

Two signals, discharge current, and accelerometer output, are displayed onto the oscilloscope screen simultaneously. And the screen image is recorded as a JPG file onto an USB memory.

Measurements are performed from cursors on the oscilloscope screen. We measure the peak discharge current value, and the peak acceleration value of the very first alternation of the oscillating accelerometer signal.

This method is required because the brief and strong propelling shock triggers the natural oscillation of the piezoelectric crystal signal, at its resonance frequency, of more than $15 \mathrm{kHz}$. An oscillation which is not what we want to measure. This is discussed into the main paper. And it triggers also Brass rod oscillation.

Therefore here, we show oscillograms and recorded measurements with few comments.

\section{E.2 Control Discharges Into Copper Discs}

Two discharges are made into the Copper discs in Configuration \#2, and with two different charge voltages (200 and 300 Volts). 


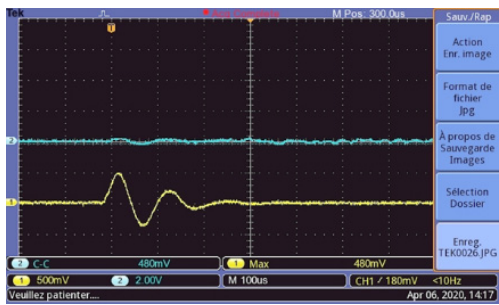

Figure E-1. Discharge of 200 Volts (4.13 Joules) into Copper discs. Channel \#1 (yellow) : Discharge current. Peak value $=2216$ Amperes. Channel \#2 (Blue) Accelerometer signal (almost nil at $10 \mathrm{~g}$ per Volt). Time $100 \mu \mathrm{s}$ per division

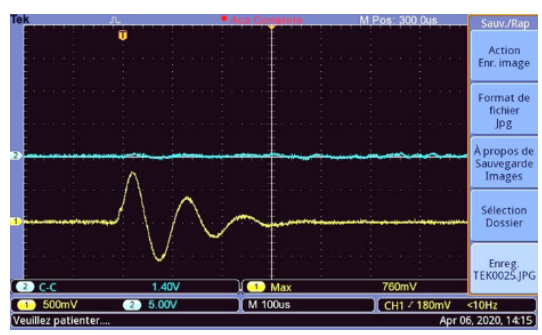

Figure E-2. Discharge of 307 Volts (9.73 Joules) into Copper discs. Channel \#1 (yellow) : Discharge current. Peak value $=3508$ Amperes. Channel \#2 (Blue) Accelerometer signal (almost nil at $10 \mathrm{~g}$ per Volt). Time $100 \mu \mathrm{s}$ per division.

The first experiments we have recorded are using a stack of two Copper discs instead of an emitter, in configuration \#2.

Copper is not superconducting at room temperature, therefore it does not contain Cooper pairs of electrons, but only free electrons. When we make an electric discharge into Copper, these free electrons are not strongly accelerated, they only move relatively slowly through the "jungle" of atoms, making numerous collisions, captures, and ionizations. No propelling effect is created.

The role of control experiments with Copper discs (Dummy emitter) is to make sure there is not any observable set of Ampere forces acting onto the powering flexible conductors that could be transmitted to the emitter support or to the accelerometer.

During these discharges, we observe a tiny electromagnetic induction during the discharge, but nothing later, only noise. The discharge current oscillates at $\sim 7700 \mathrm{Hertz}$. We also observe that the discharge current wave shape is amortized and sinusoidal. This way we are certain that there is not any artefact into the accelerometer signal.

\section{E.3 Control Shocks for the Accelerometer Signal Polarity}

Our next control experiments are about the polarity of the accelerometer signal onto the oscilloscope screen, versus the acceleration direction applied to the emitter support. This control is quite simple: a small mechanical shock is applied manually onto one of the electrodes of the emitter support while recording the accelerometer signal. For this, we used a screw driver handle, to apply the small shock to the emitter support, into successively both directions.

E.3.1 First a Shock towards Accelerometer Direction

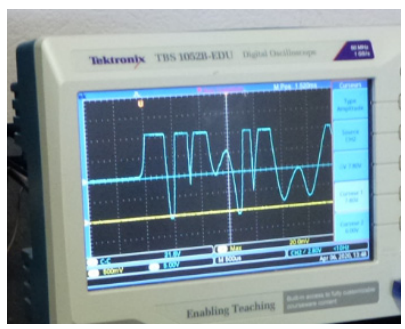

Figure E-3. Accelerometer signal oscillation, during control experiment, with manual shock on the back of the emitter support, towards the accelerometer, while recording the accelerometer signal on the oscilloscope. Time $=$ $250 \mu \mathrm{s} /$ div. POLARITY POSITIVE 
E.3.2 Then a Shock Opposed to Accelerometer Direction

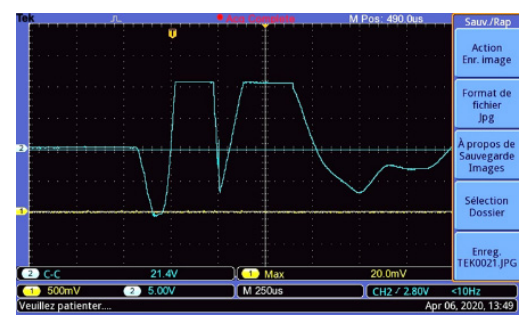

Figure E-4.upport, opposed to the accelerometer direction, while recording the accelerometer signal on the oscilloscope. Time $=250 \mu \mathrm{s} / \mathrm{div}$. POLARITY NEGATIVE

E.3.3 Then a Shock Also Opposed to Accelerometer Direction but with a Wider Time Window

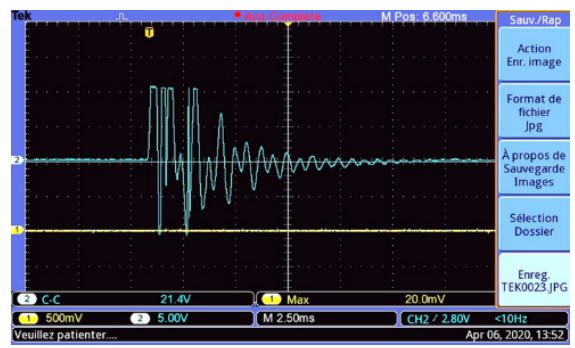

Figure E-5. Accelerometer signal oscillation, during the control experiment, with manual shock on the back of the emitter support, while recording the accelerometer signal on the oscilloscope, at a different time scale. Time $=2.5 \mathrm{~ms} / \mathrm{div}$. The full attenuation of the accelerometer oscillation needs 15 milliseconds

From figure E-5, we see that there are at least two different frequencies interferences. One at about $2.5 \mathrm{kHz}$, is the longitudinal oscillation of the Brass rod. The other one, at about $16 \mathrm{kHz}$, is the piezoelectric crystal oscillation, triggered by the brief shock. All piezoelectric accelerometers show that kind of oscillation, at high frequency, there is no way to amortize the piezoelectric crystal.

\section{E.4 Experimental Results with Emitter Em273 in Configuration \#1}

E.4.1 Discharges with MINUS Pole towards Accelerometer.

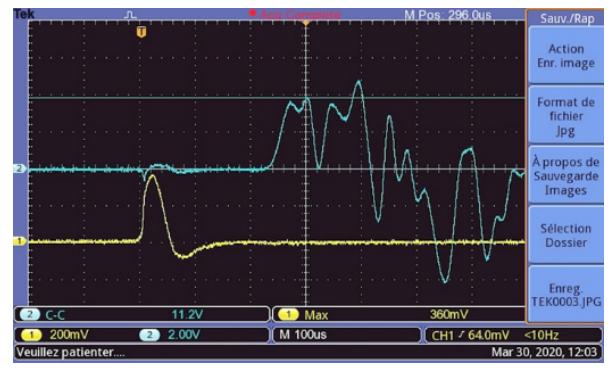

Figure E-6. Discharge of 210 Volts (4.55 Joules) into EM 273 into configuration Minus 1. Channel \#1 (yellow): Discharge current. Peak value $=1662$ Amperes. Channel \#2 (Blue) Accelerometer signal, at $10 \mathrm{~g}$ per Volt $=+$ $38.4 \mathrm{~g}$. Time $100 \mu$ s per division 


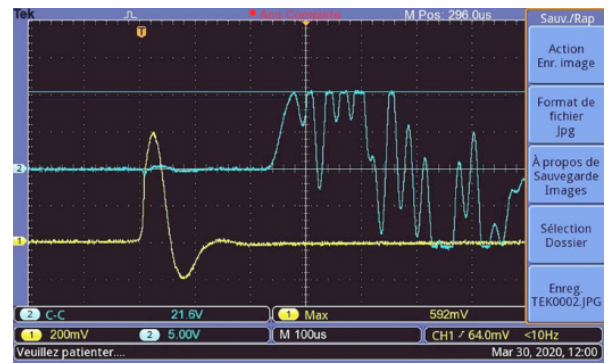

Figure E-7. Discharge of 318 Volts (10.4 Joules) into EM 273 into configuration Minus 1. Channel \#1 (yellow) : Discharge current. Peak value = 2733 Amperes. Channel \#2 (Blue) Accelerometer signal (almost saturated at 10 $\mathrm{g}$ per Volt $)=+104 \mathrm{~g}$. Time $100 \mu$ s per division.

Onto all oscilloscope screen images we are showing here, is a horizontal blue cursor line aligned with the summit of the very first accelerometer signal alternation.

It is from this cursor that we measure the peak acceleration level, at an instant where it must really be the propelling effect.

We can see effectively that the accelerometer signal begins to increase about $230 \mu \mathrm{s}$ after the beginning of the discharge current rise. This delay is the real Brass rod delay. Incidentally, we can measure the sound speed into the Brass rod: $0.623 / 230.10^{-6}=2709$ meters per second.

It is very unlikely that the propelling only acceleration value, for the 318 volts discharge, is really 104 g's (1020 $\mathrm{m} / \mathrm{s}^{2}$, which is really tremendous), because there are two mechanical oscillations superposed, this is discussed into the main paper. Another effect has to be explained.

There is also a tiny electromagnetic perturbation during the discharge, and it has no effect on the main propelling acceleration oscillation. What is clear is the fact that the first alternation polarity is positive.

E.4.2 Discharges with PLUS Pole towards Accelerometer

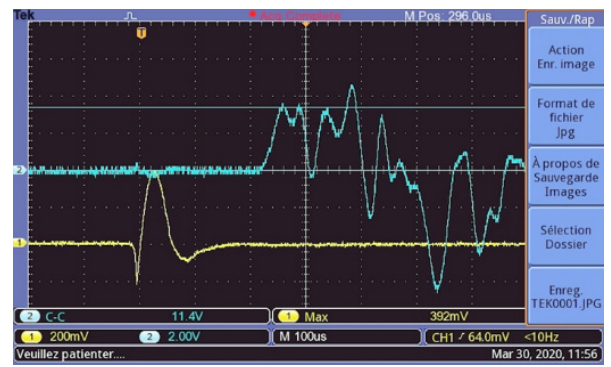

Figure E-8. Discharge of 213 Volts (4.55 Joules) into EM 273 into configuration Plus 1. Channel \#1 (yellow) : Discharge current. Peak value $=1809$ Amperes. Channel \#2 (Blue) Accelerometer signal at $10 \mathrm{~g}$ per Volt $=+$ 35,2 g. Time $100 \mu$ s per division.

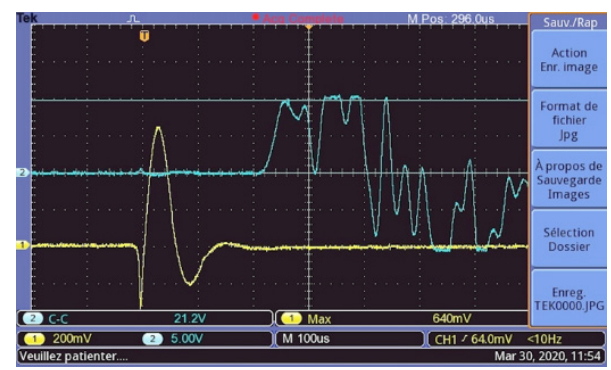

Figure E-9. Discharge of 325 Volts (10.9 Joules) into EM 273 into configuration Plus 1. Channel \#1 (yellow) : Discharge current. Peak value $=2954$ Amperes. Channel \#2 (Blue) Accelerometer signal at 10 g per Volt $=+96$ g. Time $100 \mu$ s per division. 
There is an unforeseen behavior here, which is the fact that the accelerometer signal polarity does not change, when the discharge current direction is reversed.

\section{E.5 Experimental Results with Emitter Em273 in Configuration \#2}

E.5.1 Discharges with MINUS Pole towards Accelerometer

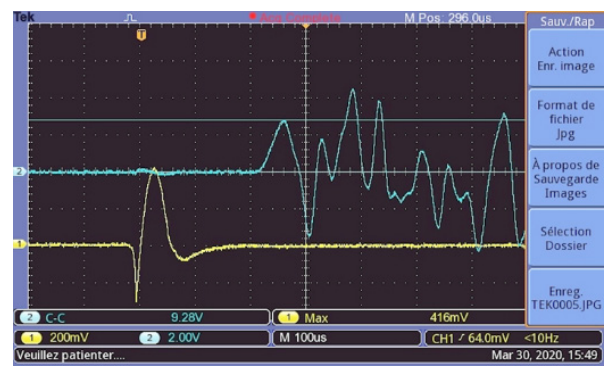

Figure E-10. Discharge of 199 Volts (4.09 Joules) into EM 273 into configuration Minus 2. Channel \#1 (yellow) : Discharge current. Peak value $=1920$ Amperes. Channel \#2 (Blue) Accelerometer signal at $10 \mathrm{~g}$ per Volt $=+28$ g. Time $100 \mu$ s per division.

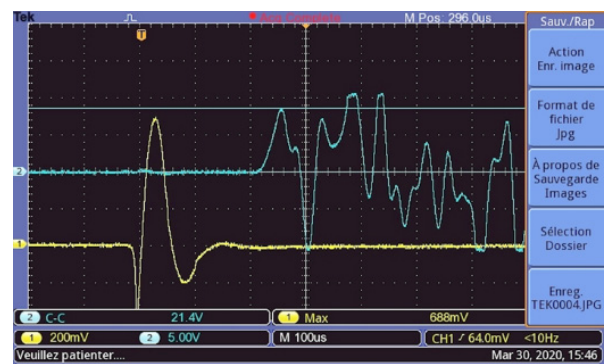

Figure E-11. Discharge of 304 Volts (9.54 Joules) into EM 273 into configuration Minus 2. Channel \#1 (yellow) : Discharge current. Peak value $=3176$ Amperes. Channel \#2 (Blue) Accelerometer signal at $10 \mathrm{~g}$ per Volt $=+86$ g. Time $100 \mu$ s per division

E.5.2 Discharges with PLUS Pole towards Accelerometer

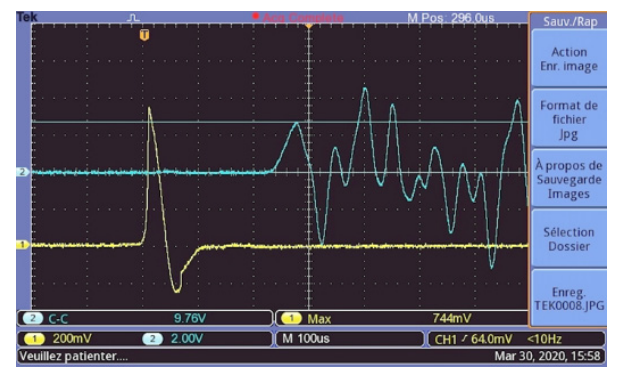

Figure E-12. Discharge of 204 Volts (4.3 Joules) into EM 273 into configuration Minus 2. Channel \#1 (yellow) : Discharge current. Peak value $=3434$ Amperes. Channel \#2 (Blue) Accelerometer signal at $10 \mathrm{~g}$ per Volt $=+$ 27.2 g. Time $100 \mu$ s per division.

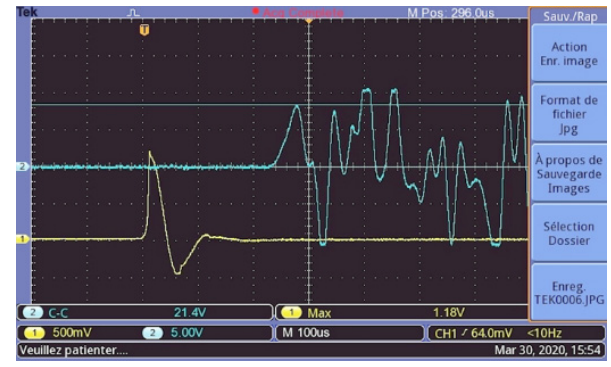


Figure E-13. Discharge of 312 Volts (10.5 Joules) into EM 273 into configuration Plus 2. Channel \#1 (yellow) : Discharge current. Peak value $=5447$ Amperes. Channel $\# 2$ (Blue) Accelerometer signal at $10 \mathrm{~g}$ per Volt $=+84$ g. Time $100 \mu$ s per division.

\section{E.6 Experimental Results with Emitter Em273 in Configuration \#3}

E.6.1 Discharges with MINUS Pole towards Accelerometer

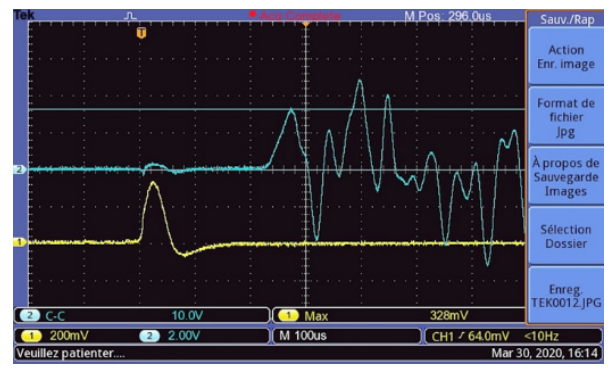

Figure E-14. Discharge of 202 Volts (4.21 Joules) into EM 273 into configuration Minus 3. Channel \#1 (yellow) : Discharge current. Peak value $=1514$ Amperes. Channel \#2 (Blue) Accelerometer signal at $10 \mathrm{~g}$ per Volt $=+32.8 \mathrm{~g}$. Time $100 \mu$ s per division.

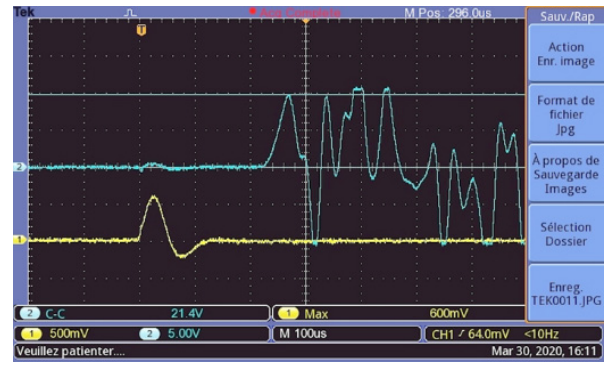

Figure E-15. Discharge of 316 Volts (10.3 Joules) into EM 273 into configuration Minus 3. Channel \#1 (yellow) : Discharge current. Peak value $=2770$ Amperes. Channel \#2 (Blue) Accelerometer signal at $10 \mathrm{~g}$ per Volt $=+98 \mathrm{~g}$. Time $100 \mu$ s per division.

E.6.2 Discharges with MINUS Pole towards Accelerometer

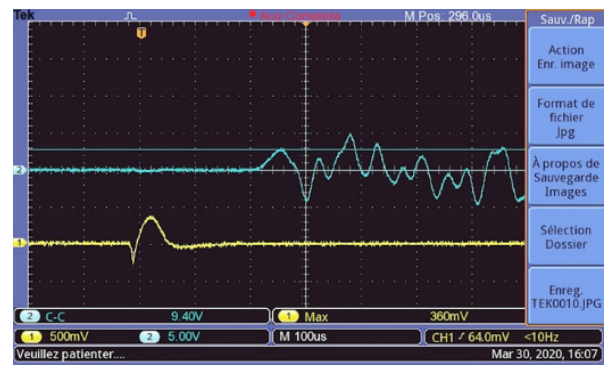

Figure E-16. Discharge of 201 Volts (4.17 Joules) into EM 273 into configuration Plus 3. Channel \#1 (yellow): Discharge current. Peak value $=1734$ Amperes. Channel \#2 (Blue) Accelerometer signal at $10 \mathrm{~g}$ per Volt $=+28$ g. Time $100 \mu$ s per division. 


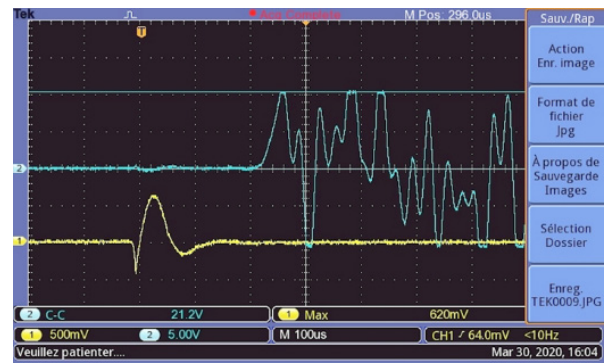

Figure E-17. Discharge of 319 Volts (10.5 Joules) into EM 273 into configuration Plus 3. Channel \#1 (yellow) : Discharge current. Peak value = 2862 Amperes. Channel \#2 (Blue) Accelerometer signal at $10 \mathrm{~g}$ per Volt $=+$ $104 \mathrm{~g}$. Time $100 \mu \mathrm{s}$ per division.

\section{E.7 Results with Emitter Em273, Turned Over, in Configuration \#2}

\section{E.7.1 Why This Experiment?}

We have observed, during all previous 12 discharges into EM273, that the first alternation of the accelerometer signal is always positive.

This means, according to our controls, that the acceleration is ALWAYS oriented towards the accelerometer direction.

Of course the first alternation amplitude varies, as expected theoretically, but there seems to be another phenomenon, that looks like a "dilatation" of the whole emitter, a systematic increase of its thickness, which varies in amplitude, with the discharge electric energy.

Therefore we need to check that the effect is effectively an expansion, this means a symmetric change of the distance between the accelerometer and the emitter center.

The method to check that, is to turn over mechanically emitter EM 273, and to replicate exactly one of the 10 Joules discharges. We chose to replicate discharge of figure E-13 in configuration \#2 after turning over the emitter into its support.

E.7.2 Discharges with PLUS Pole towards Accelerometer, to Be Compared with Figure E-13

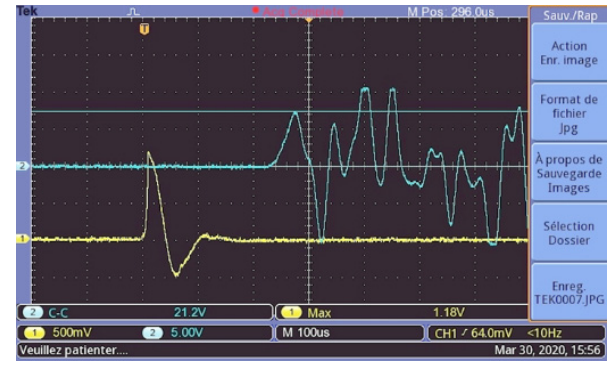

Figure E-18. Discharge of 312 Volts (10.5 Joules) into EM 273 into configuration Plus 2, With emitter turned over, (Identical to figure E-13). Channel \#1 (yellow) : Discharge current. Peak value $=5446.88$ Amperes.

Channel \#2 (Blue) Accelerometer signal at $10 \mathrm{~g}$ per Volt $=+84 \mathrm{~g}$. Time $100 \mu$ s per division.

The results are all exactly the same as into figure E-13, therefore the effect is effectively an emitter expansion (A very rapid change of thickness).

\section{E.8 Experimental Results with Emitter Em265 in Configuration \#2}

We decided to compare experimental results from EM273, with those with EM265, into configuration \#2, where there should not be any interaction with the Earth Gravity acceleration. So we use now emitter EM265 with more Graphite mass. This emitter has also more thickness, so we will see the effect of a change in internal electric fields amplitude. Emitter EM265 requires more charge voltage than EM 273 for similar accelerations. 
E.8.1 Discharges with MINUS Pole towards Accelerometer

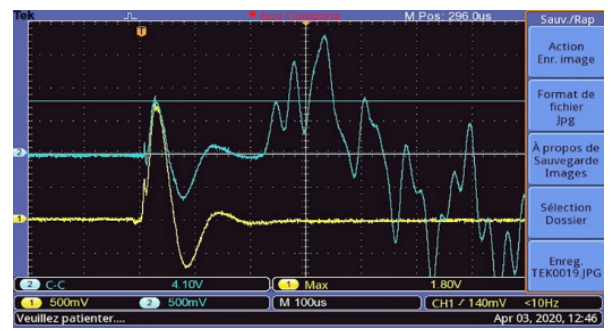

Figure E-19. Discharge of 907 Volts (84.9 Joules) into EM 265 into configuration Minus 2. Channel \#1 (yellow) : Discharge current. Peak value $=8309$ Amperes. Channel \#2 (Blue) Accelerometer signal at $1 \mathrm{~g}$ per Volt $=+0.78 \mathrm{~g}$. Time $100 \mu \mathrm{s}$ per division.

Effectively, the acceleration reduction is spectacular with emitter EM265, even with 84.9 Joules energy.

Of course, there is also much more electromagnetic perturbation, during the discharge.

Let us now double the voltage $U o$ and therefore the electric field amplitude:

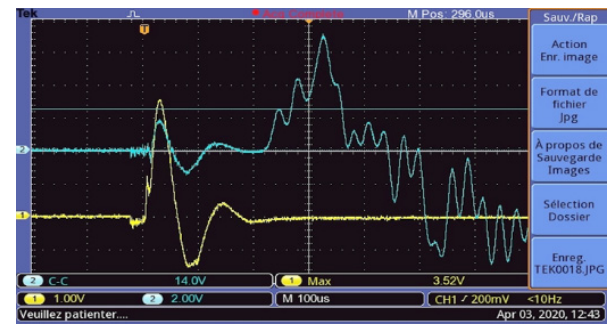

Figure E-20. Discharge of 1806 Volts (337 Joules) into EM 265 into configuration Minus 2. Channel \#1 (yellow) : Discharge current. Peak value $=15325$ Amperes. Channel \#2 (Blue) Accelerometer signal at $1 \mathrm{~g}$ per Volt $=+2.48 \mathrm{~g}$. Time $100 \mu \mathrm{s}$ per division.

E.8.2 Discharges with PLUS Pole towards Accelerometer

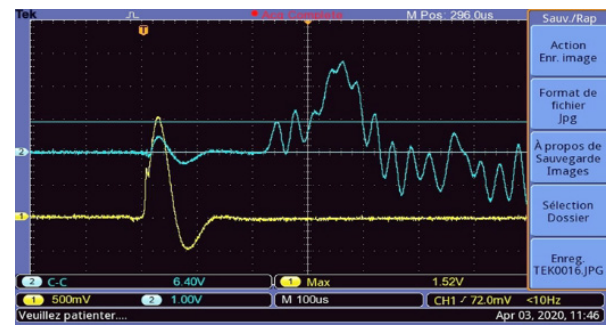

Figure E-21. Discharge of 903 Volts (84.9 Joules) into EM 265 into configuration Plus 2. Channel \#1 (yellow) : Discharge current. Peak value $=7016$ Amperes. Channel \#2 (Blue) Accelerometer signal at 1 g per Volt $=+0.92$ g. Time $100 \mu$ s per division.

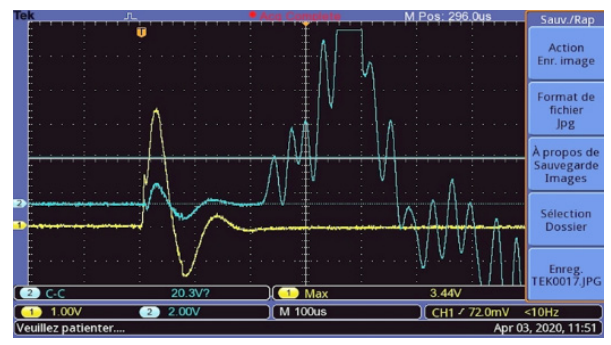

Figure E-22. Discharge of 1806 Volts (337 Joules) into EM 265 into configuration Plus 2. Channel \#1 (yellow) : Discharge current. Peak value $=15879$ Amperes. Channel \#2 (Blue) Accelerometer signal at $1 \mathrm{~g}$ per Volt $=+$ $2.72 \mathrm{~g}$. Time $100 \mu \mathrm{s}$ per division. 
Then we decided to observe a 910 Volts discharge, into EM265, at larger time scale (one millisecond per division), in order to see how the accelerometer signal oscillations attenuate.

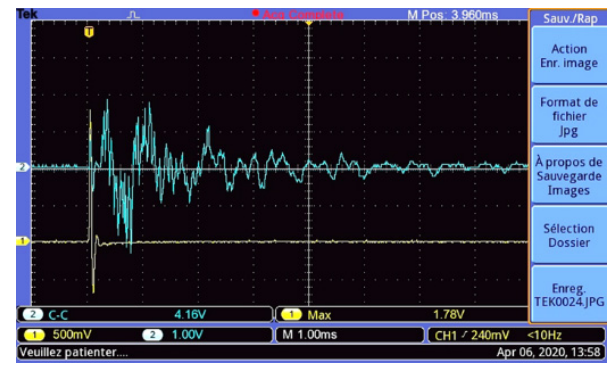

Figure E-23 — Discharge of 910 Volts (85.5 Joules) into EM 265 into configuration Plus 2. Channel \#1 (yellow) : Discharge current. Peak value $=8216$ Amperes. Channel \#2 (Blue) Accelerometer signal at $1 \mathrm{~g}$ per Volt . Time $1 \mathrm{~ms}$ per division

From E-23 results, we see that the largest amplitude, fast oscillations, are almost terminated after 3.5 milliseconds. Then the lower frequency oscillations are amortized after about 5 milliseconds, and there remains only $\pm 0.2 \mathrm{~g}$ amplitude random noise after 8 milliseconds.

\section{E.9 Is There a Thermal Expansion of Emitters?}

All results observed with EM265, rule out definitely an hypothesis of a thermal effect dilatation of the two emitters, for explaining the systematic positive polarity of the first alternation of the accelerometer signal.

Effectively, a larger graphite thickness (EM265), associated with 34 times more electric energy, should show a larger amplitude of that dilatation, by more thermal energy dissipated.

Here, instead of about $+100 \mathrm{~g}$ observed with EM 273, of $2 \mathrm{~mm}$ thickness, we have less than $+3 \mathrm{~g}$ observed with EM 265, with $15.8 \mathrm{~mm}$ graphite thickness, this with 34 times more joule effect.

So clearly the observed dilatation is a consequence of the propelling effect, not the one of a thermal effect.

To confirm that, we measured both emitters temperature, during all our discharges, and did not observed any increase of that temperature $\left( \pm<0.1^{\circ} \mathrm{C}\right)$ over the laboratory temperature at experiments time $\left(+19^{\circ} \mathrm{C}\right)$.

The emitter dilatation hypothesis does not imply a very large movement of the emitter electrodes. Effectively, a $100 \mathrm{~g}$ peak acceleration (average $50 \mathrm{~g}$ ) during $80 \mu$ s corresponds to an electrode displacement of:

$$
D=1 / 2(50.9 .81)\left(80.10^{-6}\right)^{2}=1.57 \text { micron }
$$

For a $2 \mathrm{~mm}$ thickness " $L$ " of Graphite, this corresponds to:

$$
\Delta L / L=7.85 .10^{-4}
$$

We know the dilatation coefficient of Graphite being 7.86 $.10^{-6}$ per Kelvin. Therefore the observed $\Delta L / L$ should imply a temperature increase of $\left(7.85 .10^{-4}\right) /\left(7.86 .10^{-6}\right)=99.8^{\circ} \mathrm{C}$ which would have been noticed by our thermometer, and our hands.

Moreover such a temperature increase would require hours of natural cooling down by air fins to recover the initial room temperature, not milliseconds.

\section{Therefore, no thermal effect can be incriminated.}

\section{E.10 Accelerometer Signal Spectrum}

For finishing our experiments, we measured the Fourier Spectrum of the accelerometer signal, during a 906 volts discharge into EM 265, with minus pole towards the accelerometer direction, using the Fast Fourier Transform (FFT) mode of the oscilloscope. 


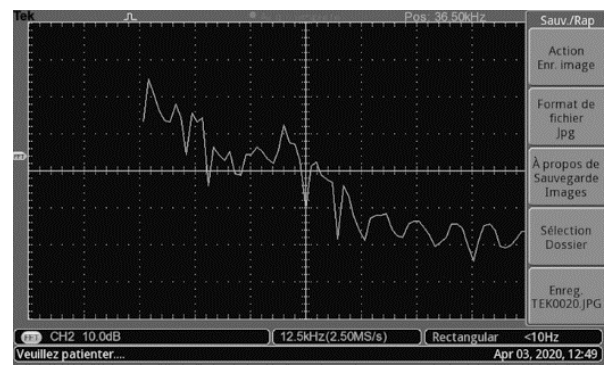

Figure E-24. Discharge of 906 Volts (84.75 Joules) into EM 265 into configuration Minus 2. Accelerometer signal spectrum, from FFT, with $10 \mathrm{~dB} /$ division vertically, and $12.5 \mathrm{kHz} /$ division horizontally.

Into figure E-24 spectrum, DC is the left end of the curve. We see that there is a first peak amplitude, at about 2.5 $\mathrm{kHz}$, which is most probably the Brass rod resonance frequency (real $2330 \mathrm{~Hz}$ ). Its amplitude is $+21 \mathrm{~dB}$. The second peak is at about $7.4 \mathrm{kHz}$, with $+12 \mathrm{~dB}$ amplitude. It could be the Propelling effect fundamental frequency component. Effectively, the discharge current oscillation frequency, during a 900 Volts discharge, (Figure E-19), is around $8000 \mathrm{Hertz}$ (period = $124 \mu \mathrm{s}$ ). After that, there is a larger peak, around $12 \mathrm{ko} 15 \mathrm{kHz}$, with amplitude +11 and $+10 \mathrm{~dB}$. This should be the piezoelectric crystal resonance oscillation (at $12.350 \mathrm{kHz}$ ). Then, farther, there are interferences frequencies, and harmonics.

The propelling signal fundamental frequency seems to be (for EM265 only and for 906 Volts only) at $+2.5 \pm 0.5$ $\mathrm{dB}$ (or $+33 \%$ amplitude) as compared to the crystal oscillating signal at $12.3 \mathrm{kHz}$.

\section{E.11 Summary of Results With Emitter Em 273}

Results from the preceding oscillograms are summarized into the following figure.

\begin{tabular}{|c|c|c|c|c|c|c|c|}
\hline $\begin{array}{c}\text { Configura- } \\
\text { tion }\end{array}$ & $\begin{array}{c}\text { Polarity } \\
\text { Towards } \\
\text { Accel. }\end{array}$ & $\begin{array}{c}\text { Charge } \\
\text { Voltage Uo } \\
\text { (Volts) }\end{array}$ & $\begin{array}{c}\text { Peak } \\
\text { current Ip } \\
\text { (Amperes) }\end{array}$ & $\begin{array}{c}\text { Peak } \\
\text { Accelera- } \\
\text { tion } \\
\text { (g) }\end{array}$ & $\begin{array}{c}\text { Current } \\
\text { waveform }\end{array}$ & $\begin{array}{c}\text { Direction } \\
\text { of Gravity } \\
\text { +owards } \\
\text { Accel }\end{array}$ & $\begin{array}{c}\text { Ratio } \\
\text { Ip/Uo } \\
\text { (Mhos) }\end{array}$ \\
\hline 1 & - & 318 & 2733 & 104 & OK & + & 8.594 \\
\hline 1 & - & 210 & 1662 & 38,4 & OK & + & 7.914 \\
\hline 1 & + & 325 & 2954 & 96 & Neg peak & + & 9.089 \\
\hline 1 & + & 213 & 1809 & 35,2 & Neg peak & + & 8.493 \\
\hline 2 & - & 304 & 3176 & 86 & Neg peak & 0 & 10.447 \\
\hline 2 & - & 199 & 1920 & 28 & Neg peak & 0 & 9.648 \\
\hline 2 & + & 312 & 5447 & 84 & Abrupt & 0 & $17.458(!)$ \\
\hline 2 & + & 204 & 3434 & 27,2 & Abrupt & 0 & $16.833(!)$ \\
\hline 3 & + & 319 & 2862 & 104 & Neg peak & - & 8.972 \\
\hline 3 & + & 201 & 1754 & 28 & Neg peak & - & 8.726 \\
\hline 3 & - & 316 & 2770 & 98 & OK & - & 8.766 \\
\hline 3 & - & 202 & 1514 & 32,8 & OK & - & 7.495 \\
\hline
\end{tabular}

Figure E-25. Results of the twelve experiments with emitter EM273, into the three configurations

\section{Copyrights}

Copyright for this article is retained by the author(s), with first publication rights granted to the journal.

This is an open-access article distributed under the terms and conditions of the Creative Commons Attribution license (http://creativecommons.org/licenses/by/4.0/). 